\title{
Optimal Configuration of 802.11e EDCA for Real-Time and Data Traffic
}

\author{
Pablo Serrano, Member, IEEE, Albert Banchs, Member, IEEE, Paul Patras, Student Member, IEEE, and \\ Arturo Azcorra, Senior Member, IEEE
}

\begin{abstract}
The enhanced distributed channel access (EDCA) mechanism of the IEEE 802.11e standard provides quality-ofservice $(\mathrm{QoS})$ support through service differentiation by using different medium-access-control (MAC) parameters for different stations. The configuration of these parameters, however, is still an open research challenge, as the standard provides only a set of fixed recommended values that do not take into account the current wireless local area network (WLAN) conditions and, therefore, lead to suboptimal performance. In this paper, we propose a novel algorithm for EDCA that, given the throughput and delay requirements of the stations that are present in the WLAN, computes the optimal configuration of the EDCA parameters. We first present a throughput and delay analysis that provides the mathematical foundation upon which our algorithm is based. This analysis is validated through simulations of different traffic sources (both data and real time) and EDCA configurations. We then propose a mechanism to derive the optimal configuration of the EDCA parameters, given a set of performance criteria for throughput and delay. We assess the effectiveness of the configuration provided by our algorithm by comparing it against 1) the recommended values by the standard, 2) the results from an exhaustive search over the parameter space, and 3) previous configuration proposals, which are both standard and nonstandard compliant. Results show that our configuration outperforms all other approaches.
\end{abstract}

Index Terms-Enhanced distributed channel access (EDCA), IEEE 802.11, performance analysis, quality-of-service (QoS), wireless local area network (WLAN).

\section{INTRODUCTION}

$\mathbf{T}$ HE IEEE 802.11e supplement [1], which is included in the new revision of the 802.11 standard [2], provides wireless local area networks (WLANs) with quality-of-service (QoS) support in the two access mechanisms specified: the enhanced distributed channel access (EDCA) and the hybrid coordination function controlled channel access. Our focus is on the former, which is an extended version of the widely supported distributed coordination function (DCF) mechanism.

Manuscript received March 17, 2009; revised August 18, 2009, November 19, 2009, and January 28, 2010; accepted January 31, 2010. Date of publication February 17, 2010; date of current version June 16, 2010. This work was supported in part by the European Community's Seventh Framework Program (FP7/2007-2013) under Grant Agreement 214994. The views and conclusions contained here are those of the authors and should not be interpreted as necessarily representing the official policies or endorsements, either expressed or implied, of the Citizens' Advanced Relationship Management Project or the European Commission. The review of this paper was coordinated by Prof. J. Li.

P. Serrano is with the Department of Telematics Engineering, University Carlos III de Madrid, 28911 Leganés, Spain (e-mail: pablo@it.uc3m.es).

A. Banchs, P. Patras, and A. Azcorra are with the Department of Telematics Engineering, University Carlos III de Madrid, 28911 Leganés, Spain, an also with the IMDEA Networks, 28912 Leganés, Spain (e-mail: banchs@it. uc3m.es; patras@it.uc3m.es; azcorra@it.uc3m.es).

Digital Object Identifier 10.1109/TVT.2010.2043274
Similarly to the DCF, the EDCA mechanism is based on the carrier sense multiple access with collision-avoidance (CSMA/CA) protocol. The main difference is that, in the new standard, different stations may contend with different values of these parameters, leading to statistical service differentiation among flows (numerical values are provided in, e.g., [3]-[6]).

When deploying an EDCA WLAN, the main challenge is the configuration of the contention parameters, as the standard provides only a set of recommended values. However, using this configuration for every scenario, regardless of, e.g., the number of stations or the traffic patterns, leads to suboptimal performance in most circumstances. Therefore, a configuration mechanism to derive the contention parameters is needed. Furthermore, this mechanism should not be based on heuristics but rather on an analytical model that provides strong mathematical foundations to guarantee optimal performance.

In this paper, we build upon our previous work to achieve a twofold objective.

- First, we present a novel analytical model of EDCA performance that accounts for generic saturated and nonsaturated sources, and provides the average throughput, the average delay, and the standard deviation of the delay as performance figures. To our knowledge, this is the most complete model of EDCA proposed to date and the only one that has all these features.

- Second, we use this new analytical model to develop a configuration mechanism for the parameters of EDCA that, taking as input the traffic requirements from both realtime and nonreal-time stations, outputs the configuration that maximizes performance: It admits as many real-time traffic stations as possible while optimizing nonreal-time throughput. To the best of our knowledge, this is the first approach to configuring EDCA that covers all traffic types and is sustained analytically, thereby guaranteeing optimal performance. $^{1}$

The rest of this paper is structured as follows. In Section II, we review the state of the art. In Section III, we describe our analytical model and validate it through exhaustive simulations. The optimal configuration mechanism is introduced in Section IV, along with the results from the numerical search to prove the effectiveness of our algorithm as well as a comparison against previous approaches. Finally, concluding remarks are given in Section $\mathrm{V}$.

${ }^{1}$ Note that the analytical model requires a series of assumptions. Therefore, when we use the term "optimal configuration," we are referring to the configuration that provides the best performance according to this model. 


\section{StATE OF THE ART}

Here, we present the state of the art. We first summarize the behavior of the EDCA mechanism, and then, we review previous analyses and approaches to configure EDCA.

\section{A. IEEE 802.11e EDCA}

Here, we briefly summarize the EDCA mechanism as defined in the 802.11e standard. EDCA is a CSMA/CA-based protocol that extends the DCF by means of the parameters that are used to access the channel. The channel access is regulated by the channel-access functions (CAFs). To transmit its frames, each $\mathrm{CAF}$ executes an independent back-off process that is regulated by a number of configurable parameters. For the configuration of these parameters, the standard groups the CAFs by access categories (ACs) and assigns the same configuration to all the CAFs of an AC. In this paper, we assume, for simplicity, that each station runs only one $\mathrm{CAF}$, and therefore, we interchangeably use the terms CAF and station. ${ }^{2}$

A station of an access category $i(A C i)$ with a new frame to transmit monitors the channel activity. If the channel is sensed idle for a period of time that is equal to the arbitration interframe space parameter of this $\mathrm{AC}\left(A I F S_{i}\right)$, the station transmits. Otherwise, if the channel is sensed busy (either immediately or during the $A I F S_{i}$ period), the station continues to monitor the channel until it is measured idle for an $A I F S_{i}$ time, and at this point, the back-off process starts. The arbitration interframe space $A I F S_{i}$ takes a value of the form DIFS+ $n T_{e}$, where DIFS and $T_{e}$ are constants that are dependent on the physical layer, and $n$ is a nonnegative integer. ${ }^{3}$

Upon starting the back-off process, the station computes a random value that is uniformly distributed in the range $\left(0, C W_{i}-1\right)$ and initializes its back-off time counter with this value. The $C W_{i}$ value is called the contention window and depends on the number of transmission attempts for the current frame. At the first transmission attempt, $C W_{i}$ is set to be equal to the minimum contention window parameter $\left(C W_{i}^{\min }\right)$. As long as the channel is sensed idle, the back-off time counter is decremented once for each time interval $T_{e}$.

When a transmission is detected on the channel, the backoff time counter is "frozen" and reactivated again after the channel is sensed idle for a certain period. This period is equal to $A I F S_{i}$ if the transmission is received with a correct frame check sequence (FCS). Otherwise, this period is equal to $E I F S-D I F S+A I F S_{i}$, where EIFS is another constant that is dependent on the physical layer.

As soon as the back-off time counter reaches zero, the station transmits its frame in the next slot time. A collision occurs when two or more stations simultaneously start a transmission. An acknowledgment (Ack) frame is used to notify the transmitting station that the frame has been successfully received. If the Ack

\footnotetext{
${ }^{2}$ Note that, following [7], the analysis here could easily be extended to the case of multiple CAFs per station.

${ }^{3}$ According to the IEEE 802.11e standard terminology, AIF $S_{i}=S I F S+$ $n T_{e}$, where $D I F S=S I F S+2 T_{e}$, and $n \geq 2$. Without loss of generality, in this paper, we use the simplified notation $\bar{A} I F S_{i}=D I F S+n T_{e}$, with $n \geq 0$.
}

is not received within a timeout, the station assumes that the frame was not received and reschedules the transmission by reentering the back-off process. After each unsuccessful transmission, $C W_{i}$ is doubled, up to a maximum value that is given by the $C W_{i}^{\max }$ parameter. If the number of failed attempts reaches a predetermined retry limit $R$, the frame is discarded.

When the station gains access to the channel, it is allowed to retain the right to access it for a duration that is equal to the transmission opportunity limit parameter $\left(T X O P_{i}\right)$. Note that the impact of the $T X O P_{i}$ parameter is typically small in QoS-provisioned scenarios, as real-time traffic parameters are usually set such that queues never grow above one packet, whereas for data traffic, this parameter is set such that only one packet is transmitted upon accessing the channel to avoid degrading the delay performance of real-time traffic. Following this reasoning, in the rest of this paper, we concentrate on the analysis of the other three parameters, i.e., $C W_{i}^{\min }, C W_{i}^{\max }$, and $A I F S_{i}$.

\section{B. Related Work}

There are several analytical models of EDCA performance available in the literature [7]-[20]. However, most of them [7][14] are based on the unrealistic assumption that all stations always have packets that are ready for transmission (commonly referred to as saturation conditions). While this assumption may be reasonable for data traffic, it does not hold for realtime traffic. On the other hand, previous approaches assuming nonsaturated conditions [15]-[20] are typically valid only for Poisson arrivals and fixed length packets. In contrast to these previous papers, our analysis makes no assumptions about the arrival process and allows for variable packet lengths.

The analysis presented in this paper combines and extends our previous work, providing the most comprehensive analysis of EDCA to date, including generic traffic sources as well as the relevant metrics for data and real-time traffic (namely, the throughput, the average, and the standard deviation of the delay). In particular, the analysis extends our previous work as follows.

- In [20], we presented an analysis of EDCA under nonsaturated traffic conditions to model the throughput and the average delay. In this paper, we also account for the standard deviation of the delay.

- In [13], we analyzed the average delay performance of EDCA. While [13] is limited to saturation conditions, the present analysis also considers nonsaturation traffic.

- In [21], we analyzed the standard delay deviation when there is only voice traffic that is present in the WLAN. In this paper, we extend this analysis to the case where there are multiple ACs.

The differences between the model presented in this paper and the previous work are summarized in Table I. We observe that the proposed model is more complete than any of the previous models.

Only recently has the challenge of configuring the EDCA parameters been addressed [7], [21]-[27]. However, the existing approaches suffer from major drawbacks. Our previous works in [7] and [22] are restricted to data traffic, whereas our works 
TABLE I

Comparison Between the Analytical Models of EDCA Performance

\begin{tabular}{|l|c|c|c|c|c|c|c|c|}
\hline & Ours & [7]-[11] & [12], [13] & [17], [19] & [15] & [16], [18] & [20] & [21] \\
\hline \hline CW & $\checkmark$ & $\checkmark$ & $\checkmark$ & $\checkmark$ & $\checkmark$ & $\checkmark$ & $\checkmark$ & $\checkmark$ \\
AIFS & $\checkmark$ & $\checkmark$ & $\checkmark$ & $\cdot$ & $\checkmark$ &. & $\checkmark$ &. \\
Average Delay & $\checkmark$ & $\cdot$ & $\checkmark$ & $\checkmark$ & $\checkmark$ & $\cdot$ & $\checkmark$ & $\checkmark$ \\
Standard Dev & $\checkmark$ & $\cdot$ & $\cdot$ & $\cdot$ & $\cdot$ & $\cdot$ & $\cdot$ & $\checkmark$ \\
Non-saturation & $\checkmark$ & $\cdot$ & $\cdot$ & $\checkmark$ & $\checkmark$ & $\checkmark$ & $\checkmark$ & $\checkmark$ \\
Generic sources & $\checkmark$ & $\cdot$ & $\cdot$ & $\cdot$ & $\cdot$ &. & $\checkmark$ &. \\
\hline
\end{tabular}

in [21] and [23] are restricted to voice traffic. The works in [24] and [25] only consider two traffic types, i.e., voice and data, and do not allow different types of real-time and nonreal-time ${ }^{4}$ traffic. The default configuration recommended by the standard [2], the one recommended in [26], and the adaptive mechanism in [27] consider all traffic types, but they are based on heuristics and, therefore, do not guarantee optimal performance. Indeed, the performance evaluation conducted shows that our proposal substantially outperforms these previous proposals.

In addition to the above, a number of modifications of the EDCA protocol have recently been proposed [28]-[32]. These proposals have the major drawback of not being standardcompliant and requiring modifications to the hardware and the firmware of the wireless cards, which challenges their practical deployment. The proposal in [28] applies only to one AC, whereas the one in [29] supports only voice and best effort traffic. The approach proposed in [30] prevents data stations from transmitting when the contention level exceeds a certain threshold, which has the shortcoming of starving them. Finally, the approaches in [31] and [32] are based on heuristics; our simulation results show that our approach, even without introducing modifications to EDCA, clearly outperforms them.

\section{Performance AnAlysis}

Here, we consider a WLAN operating under the EDCA mechanism and analyze the throughput and the delay of each AC in the WLAN.

\section{A. Definitions, Terminology, and Assumptions}

In the following, we present the key definitions, terminology, and assumptions upon which our analysis is based. A summary of the notation and variables used in the analysis is provided in Table II. In particular, our analytical model takes the following input variables:

- the number of ACs in the WLAN $(N)$;

- the number of stations of each $\mathrm{AC}\left(n_{i}\right.$ is the number of stations of $A C i$ );

- the average sending rate of the stations of each $\mathrm{AC}\left(\rho_{i}\right)$, their frame length distribution, and the average frame length $\left(l_{i}\right)$;

- the configuration $\left\{C W_{i}^{\min }, m_{i}, A_{i}\right\}$ of each $\mathrm{AC}$, where $m_{i}$ is defined such that $C W_{i}^{\max }=2^{m_{i}} C W_{i}^{\min }$, and $A_{i}$ such that $A I F S_{i}=D I F S+A_{i} T_{e}$;

\footnotetext{
${ }^{4}$ Throughout this paper, we will interchangeably use the terms "data" and "nonreal time."
}

TABLE II

Notation UsED IN THE ANALysis

\begin{tabular}{|l|l|}
\hline Variable & Description \\
\hline$N$ & Number of ACs \\
$n_{i}$ & Number of stations of $A C i$ \\
$l_{i}$ & Average length of frames from $A C i$ \\
$A$ & Largest $A_{i}$ in the WLAN \\
$\rho_{i}$ & Average sending rate of $A C i$ \\
$\tau_{i}$ & Transmission probability of a station of $A C i$ \\
$r_{i}$ & Throughput of a station of $A C i$ \\
$\Delta_{k}$ & Set of ACs with $A_{i} \leq k$ \\
$p\left(\Delta_{k}\right)$ & Prob. that in a slot only set $\Delta_{k}$ can transmit \\
$p\left(t_{k}\right)$ & given only the set $\Delta_{k}$ can transmit \\
$p\left(e \mid t_{k}\right)$ & Probability that a slot is a $k$-slot \\
$p(e)$ & Prob. that a $k$-slot is empty \\
$p\left(s_{i}\right)$ & Prob. that a slot is empty \\
$p(c)$ & Prob. that a slot contains a success of $A C i$ \\
$p\left(c_{i}\right)$ & Prob. that a slot contains a collision \\
$p(s)$ & Collision prob. of an attempt of $A C i$ \\
$p\left(s_{i} \mid \Delta_{k}\right)$ & Prob. that a slot contains a success \\
& Prob. that a slot contains a success of $A C i$ \\
\hline
\end{tabular}

and provides as output the throughput, the average delay, and the standard deviation for each AC.

Note that our model can be applied to analyze generic source models. The only restriction imposed on the sources is that they are ergodic; otherwise, the analysis could not rely on the stations' average sending rate.

Our analysis is based on the following definitions.

Definition 1: A slot time is the time interval between two consecutive back-off counter decrements of a station with minimal $A I F S_{i}$ (i.e., $D I F S$ ). We say that a slot time is nonempty when it contains a collision or a successful transmission and that it is empty otherwise.

Definition 2: A slot time is a $k$-slot time if it is preceded by $k$ or more empty slot times.

Definition 3: The saturation rate of an $\mathrm{AC}$ is the rate that the stations of this $\mathrm{AC}$ would obtain if they always had a packet that is ready for transmission.

Based on these definitions, our analysis relies on a number of assumptions. First, we make the following two key approximations around the notion of saturation rate to compute the stations' rates in the WLAN.

- As long as the average sending rate of the stations of a given $\mathrm{AC}$ falls below their saturation rate, we assume that the stations of this $\mathrm{AC}$ see all their packets served (i.e., their transmission queue never overflows). We refer to such an $\mathrm{AC}$ as a nonsaturated $\mathrm{AC}$.

- On the other hand, if the average sending rate of the stations of the $\mathrm{AC}$ exceeds the saturation rate, we consider that the stations of this $\mathrm{AC}$ always have packets that are ready for transmission (i.e., their transmission queue never empties). We refer to such an AC as saturated. 


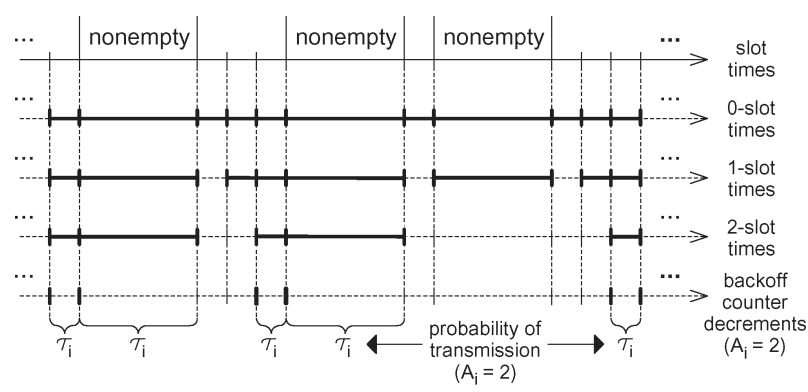

Fig. 1. $k$-slot times and probability of transmission (example with $k=2$ ).

In addition to the above two approximations, our analysis further relies on the following additional assumptions that have already been used in previous works in the literature.

- Back-off times follow a geometric distribution (i.e., a station transmits upon decrementing its counter with an independent probability). This assumption was first used in the analysis in [33] for the 802.11 DCF, and since then, it has been used in most of the analyses of EDCA (see, e.g., [8]-[10]). Although back-off times actually follow a uniform distribution, all these works have shown that this assumption leads to accurate results.

- Each packet transmission attempt collides with an independent probability. This assumption, which is initially used in [34], has been the basis of most of the WLAN performance analyses so far.

- The length of a slot time can be modeled with a random variable that depends only on the stations that could potentially transmit in this slot time. This is also a common assumption when analyzing the delay performance of EDCA (see, e.g., [11]).

- Finally, at each transmission attempt, the packet length follows a random variable that depends only on the considered $A C i$. This assumption is necessary for the tractability of the analysis and has been used and shown to be accurate in previous analyses dealing with variable packet lengths in WLANs (see, e.g., [20] and [34]).

We build our analysis upon the variable $\tau_{i}$, which is defined as the probability that a station of $A C i$ transmits upon a backoff counter decrement. Note that since a station with $A_{i}=k$ starts decrementing its back-off counter only after $k$ empty slot times following a nonempty slot time, we see that the back-off counter decrements of this station coincide with the boundaries of the $k$-slot times. Therefore, a station of $A C i$, with $A_{i}=$ $k$, transmits in a $k$-slot time with probability $\tau_{i}$ and does not transmit in any other slot time (see Fig. 1).

In the following, we first separately analyze the $\tau_{i}$ of a saturated $\mathrm{AC}$ and the $\tau_{i}$ of a nonsaturated $\mathrm{AC}$ and combine both analyses to compute the $\tau_{i}$ values of all the ACs in the WLAN. Then, based on these values, we calculate the throughput and delay performance of each AC.

\section{B. Point of Operation of the WLAN}

We first compute the point of operation of the WLAN as given by the transmission probabilities $\tau_{i}$ 's of all the ACs. We start with the case of a saturated AC [13]. With the assumption that each transmission attempt collides with a constant and independent probability, we can model the behavior of this AC with the same Markov chain as in [35, Fig. 5]. Then, the probability that a station of a saturated AC transmits upon a back-off counter decrement can be computed by means of (1), shown at the bottom of the page, which is given by [35], where $p\left(c_{i}\right)$ is the probability that a transmission attempt of a station of $A C i$ collides.

We next focus on the analysis of a nonsaturated AC. The goal of this analysis is to compute the probability that a nonsaturated station transmits in a slot time, i.e., $\tau_{i}^{\text {nonsat }}$. Note that, in contrast to the $\tau_{i}$ of a saturated station, which exclusively depends on the back-off process, $\tau_{i}^{\text {nonsat }}$ also accounts for the inactivity periods of the station caused by its queue being empty. The following lemma lets us compute the $\tau$ of a nonsaturated AC based on variables that, as shown in the Appendix, can be expressed as a function of $\tau_{i}$ 's and $p\left(c_{i}\right)$ 's.

Lemma 1: The $\tau_{i}$ of a nonsaturated AC is given by ${ }^{5}$

$$
\tau_{i}^{\text {nonsat }}=\frac{\rho_{i}\left(1-p\left(c_{i}\right)^{R+1}\right)\left(p(s) T_{s}+p(e) T_{e}+p(c) T_{c}\right)}{l_{i}\left(1-\tau_{i}\right)^{n_{i}-1} \sum_{k=A_{i}}^{A} p\left(\Delta_{k}\right) \prod_{j \in \Delta_{k} \backslash i}\left(1-\tau_{j}\right)^{n_{j}}}
$$

where $p(s), p(c)$, and $p(e)$ are the probabilities that a slot time contains a successful transmission, contains a collision, or is empty, respectively, and $T_{s}, T_{c}$, and $T_{e}$ are the average slottime durations in each case. $\Delta_{k}$ is the set of ACs with $A_{i} \leq k$, and $p\left(\Delta_{k}\right)$ is the probability that a randomly chosen slot time is allowed for transmission to the set $\Delta_{k}$.

With the above, we can express $\tau_{i}$ 's as a function of the rest of the $\tau_{i}$ 's and $p\left(c_{i}\right)$. To build a system of equations, we need to express $p\left(c_{i}\right)$ as a function of the rest of the $\tau_{i}$ 's. We compute $p\left(c_{i}\right)$ as a function of the probability of an empty $k$-slot time [which is denoted by $p\left(e \mid t_{k}\right)$ ] as follows. A $k$-slot time is empty as long as 1) the considered station does not transmit, and 2) no other station transmits. The latter can be expressed as a function of $p\left(c_{i}\right)$ by noting that the probability of a collision corresponds to the case when some other station transmits. Thus

$$
p\left(e \mid t_{k}\right)=\left(1-\tau_{i}\right)\left(1-p\left(c_{i}\right)\right)
$$

which yields

$$
p\left(c_{i}\right)=1-\frac{p\left(e \mid t_{k}\right)}{1-\tau_{i}}
$$

\footnotetext{
${ }^{5}$ The proofs of all lemmas are derived in the Appendix.
}

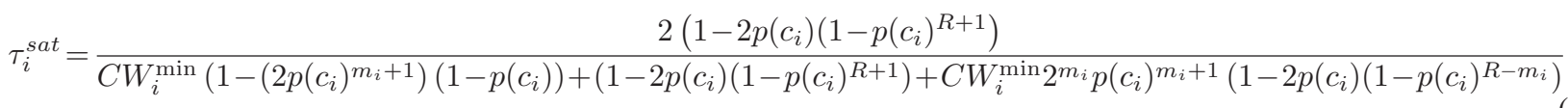




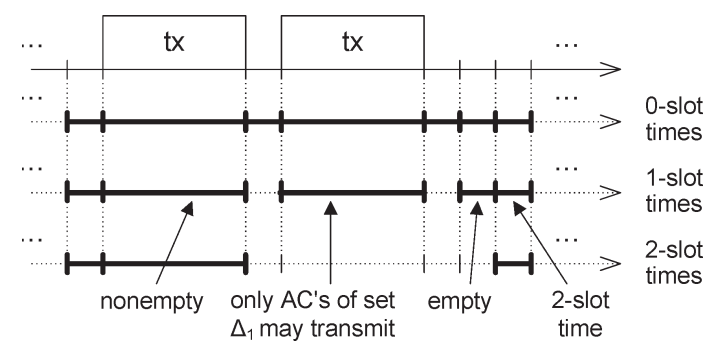

Fig. 2. Probability of an empty $k$-slot time (example with $k=1$ ).

Now, let us focus on the probability that a given $k$-slot time is empty. If the previous $k$-slot time was nonempty, in this $k$-slot time, only the ACs with $A_{i} \leq k$ may transmit. If the previous $k$-slot time was empty, the given $k$-slot time is preceded by $k+1$ or more empty slot times, which is exactly the definition of $(k+1)$-slot time, and therefore, such a $k$-slot time is empty with probability $p\left(e \mid t_{k+1}\right)$. Applying this reasoning (see Fig. 2), $p\left(e \mid t_{k}\right)$ can be written as

$$
p\left(e \mid t_{k}\right)=\left(1-p\left(e \mid t_{k}\right)\right) \prod_{j \in \Delta_{k}}\left(1-\tau_{j}\right)^{n_{j}}+p\left(e \mid t_{k}\right) p\left(e \mid t_{k+1}\right) .
$$

Note that, if $A$ is the largest $A_{i}$ in the WLAN, in a $A$-slot time, all stations may transmit; therefore, the following holds:

$$
p\left(e \mid t_{A}\right)=\prod_{j \in \Delta_{A}}\left(1-\tau_{j}\right)^{n_{j}} .
$$

Starting from $\tau_{i} \forall i$, with (6), we can compute $p\left(e \mid t_{A}\right)$. Then, with (5), we can compute $p\left(e \mid t_{A-1}\right)$. Applying this recursively, we can compute $p\left(e \mid t_{k}\right) \forall k$. Then, $p\left(c_{i}\right)$ can be computed using (4), and finally, $\tau_{i}$ can be obtained from (1).

We next combine the analyses for a saturated and a nonsaturated $A C$ to obtain all $\tau_{i}$ 's in the WLAN under stationary conditions. From the above, we have a method to compute the $\tau_{i}$ of a saturated and of a nonsaturated AC; the remaining challenge lies in determining which ACs are saturated and which are not. For this purpose, we proceed step by step as follows.

- In the first step, we consider that all ACs are saturated. Note that, from (1) and (2), we can express each $\tau_{i}$ of a saturated (or nonsaturated) AC as a function of all $\tau_{i}$ 's. Therefore, we have a system of $N$ nonlinear equations on $\tau_{i}$ 's that can be resolved using numerical techniques. Once the $\tau_{i}$ values have been derived, we compute the throughput of all ACs by using Lemma 2. We next compare the throughputs against the sending rates. If the throughput of an $\mathrm{AC}$ is larger than its sending rate, we consider from this step on that this $\mathrm{AC}$ is not saturated and move it to the set of nonsaturated ACs.

- In the second step, we take the new sets of saturated and nonsaturated ACs resulting from the first step and repeat the throughput computation. Next, we again compare the throughputs obtained in the previous step for the saturated ACs against their sending rates and move those ACs whose throughputs are larger than their sending rates to the set of nonsaturated ACs. ${ }^{6}$

- The above is iteratively done until the resulting throughputs of all the saturated ACs are smaller than their sending rates. This last scenario represents a stable solution, and therefore, the values from this step give us the throughput that each AC will obtain in the WLAN under stationary conditions.

Note that, as the number of ACs $N$ is limited to four by the standard, the above procedure requires, in the worst case, that we resolve, at most four times, a system of no more than four equations, and therefore, the computational complexity is low.

\section{Throughput and Delay Analysis}

Once the values $\tau_{i}$ 's have been derived, we can analyze the throughput and the delay performance of the WLAN. More specifically, in the following, we analyze the average throughput, the average service delay, and the standard deviation of the delay. $^{7}$

The throughput $r_{i}$ is given by the following lemma.

Lemma 2: The average throughput $r_{i}$ that a station from $A C i$ experiences is given by

$$
r_{i}=\frac{l_{i} \sum_{k=A_{i}}^{A} p\left(\Delta_{k}\right) p\left(s_{i} \mid \Delta_{k}\right)}{(s) T_{s}+p(c) T_{c}+p(e) T_{e}}
$$

where $p\left(s_{i} \mid \Delta_{k}\right)$ is the probability that, given that only stations from set $\Delta_{k}$ can transmit, there is a successful transmission from $A C i$.

We next compute the delay performance of the WLAN. For this purpose, we define $B_{i, r}$ as the average back-off counter before retry $r, T_{\text {slot, } k}^{i}$ as the average duration of a $k$-slot time in which the considered station of $A C i$ does not transmit, $T_{\text {inter } \_t x, k}^{i}$ and $T_{\text {inter, } k}^{i}$ as the average durations of the time between two $k$-slot times when the considered station transmits and does not transmit in the first one, respectively, and $T_{s, i}$ and $T_{c, i}$ as the average durations of a slot time that contains a success and a collision involving a station of $A C i$. In Figs. 3 and 4, we illustrate these delay components for a given sequence of slot times. Based on these variables, Lemma 3 provides the average value of the delay $d_{i}$.

Lemma 3: The average delay experienced by a nondropped packet of a station of $A C i$ is given by

$$
\begin{aligned}
d_{i} & =\frac{1}{\sum_{j=0}^{R}\left(1-p\left(c_{i}\right)\right) p\left(c_{i}\right)^{j}} \sum_{j=0}^{R}\left(1-p\left(c_{i}\right)\right) p\left(c_{i}\right)^{j} \\
& \times\left(j T_{c, i}+T_{s, i}+\sum_{r=0}^{j}\left(T_{\text {inter_tx }, k}^{i}+B_{i, r}\left(T_{\text {slot }, k}^{i}+T_{\text {inter }, k}^{i}\right)\right)\right) .
\end{aligned}
$$

${ }^{6}$ Note that an $\mathrm{AC}$ that was not saturated in the previous step can never become saturated again. In fact, if such an AC always had packets that are ready for transmission, it would obtain a throughput that is even larger than in the step where it became nonsaturated (since in the current step, there are fewer saturated ACs).

${ }^{7}$ Given the average delay and its standard deviation, it is possible to provide guarantees on the delay distribution by means of the Chebyshev inequality [36]. In this paper, we do not discuss this further and simply assume that the average delay and the standard deviation are sufficient to provide real-time traffic with the desired service guarantees. 


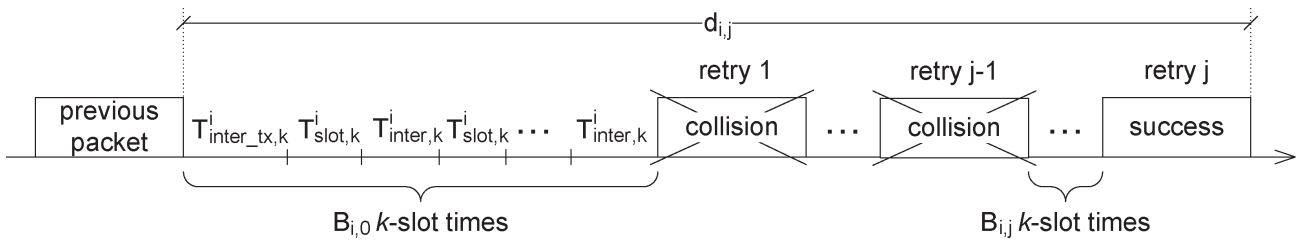

Fig. 3. Average delay components for the case of $j$ retries.

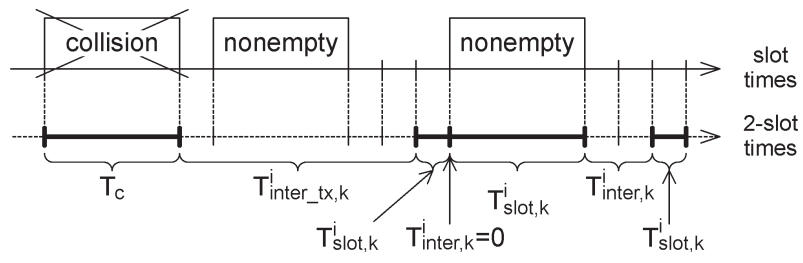

Fig. 4. Components of the delay (example with $k=2$ ).

Finally, the following lemma gives the value of the standard deviation of the delay.

Lemma 4: The standard deviation of the delay is given by

$$
\sigma_{d_{i}}^{2}=\frac{\sum_{j=0}^{R}\left(1-p\left(c_{i}\right)\right) p\left(c_{i}\right)^{j} E\left[\left(d_{i, j}\right)^{2}\right]}{\sum_{j=0}^{R}\left(1-p\left(c_{i}\right)\right) p\left(c_{i}\right)^{j}}-\left(d_{i}\right)^{2}
$$

where $d_{i, j}$ is the delay that a frame from $A C i$ suffers in case of $j$ retries.

The above lemma terminates our performance analysis of EDCA. Section III-D is devoted to the assessment of its accuracy under different traffic sources and EDCA configurations.

\section{Performance Analysis Validation}

We validate the accuracy of the model by comparing the analytical values against those obtained by means of simulations. For this purpose, we have implemented the 802.11e EDCA protocol in OMNeT++. ${ }^{8}$ The source code of our simulations is available in our Web site. ${ }^{9}$ The simulations are performed for a WLAN with the medium-access-control (MAC) layer parameters of IEEE 802.11b [37]. We assume a channel in which frames are only lost due to collisions. The queue size of all of the stations is set equal to 100 packets. For the simulation results, the average and $95 \%$ confidence interval values are given, although, in many cases, confidence intervals are too small to be appreciated in the graphs. The values that are analytically obtained are plotted with lines, and the simulation results are plotted with points.

1) Data Traffic: First, we analyze our throughput model for the case when only data traffic is present in the network, with no delay requirements. We have taken a fixed frame payload size of $1500 \mathrm{~B}$, and $m_{i}=5$ (i.e., $C W_{i}^{\max }=2^{5} C W_{i}^{\min }$ ).

We consider a scenario with four ACs, i.e., $i \in\{1, \ldots, 4\}$, with $n_{i}=2$ stations each, sharing the channel with a different $C W_{i}^{\min }$ and $A I F S_{i}$ each. Specifically, we take $C W_{i}^{\min }=2^{i-1} C W_{1}^{\min }$ for $i \in\{2,3,4\}$ and $A_{i}=i-1$ for $i \in\{1, \ldots, 4\}$. Results are given in Fig. 5. The simulations

\footnotetext{
${ }^{8}$ http://www.omnetpp.org.

${ }^{9}$ http://enjambre.it.uc3m.es/ ppatras/owsim/.
}

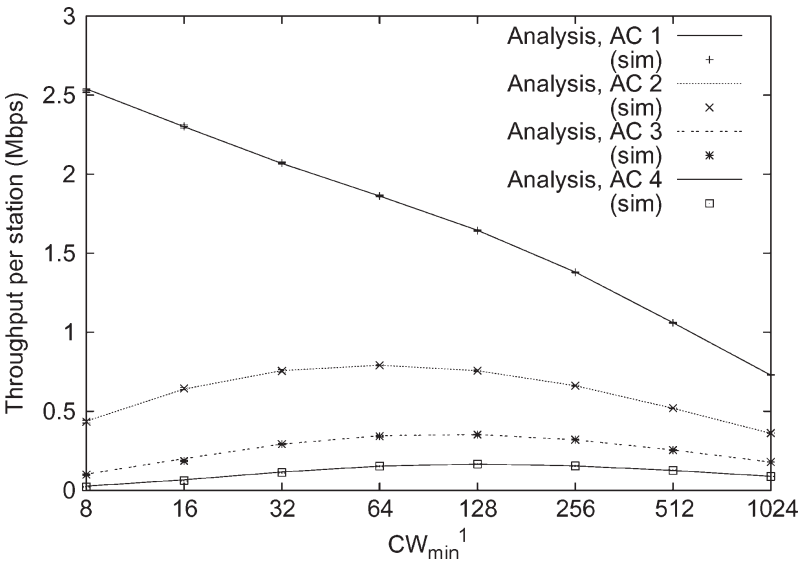

Fig. 5. Validation of the throughput model for a scenario with four ACs, each using a different $A I F S$ and $C W$ configuration.

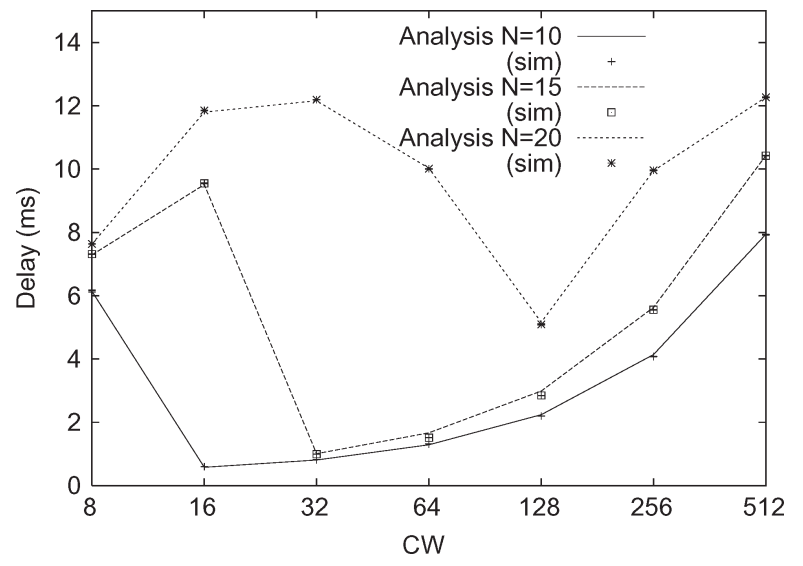

Fig. 6. Validation of the average delay model for a voice traffic scenario with different configurations of the $C W$ used.

performed validate our model for data traffic, as simulation results match the analytical ones well.

2) Voice Traffic: Next, we validate the accuracy of our analysis by comparing analytical results against simulations in a scenario where only voice traffic is present. Following the behavior of standard pulse-code modulation codecs (e.g., G.711), voice sources generate one 80 -B packet every $10 \mathrm{~ms}$.

Figs. 6 and 7 plot the average and the standard deviation of the delay, respectively, for different configurations of the $C W_{\min }$ parameter and different numbers of voice stations. The three values that are chosen for the number of voice stations $n \in\{10,15,20\}$ correspond to a low, medium, and heavily loaded WLAN, respectively. We observe that the analytical results match the simulations remarkably well, which confirms the accuracy of our analysis. 


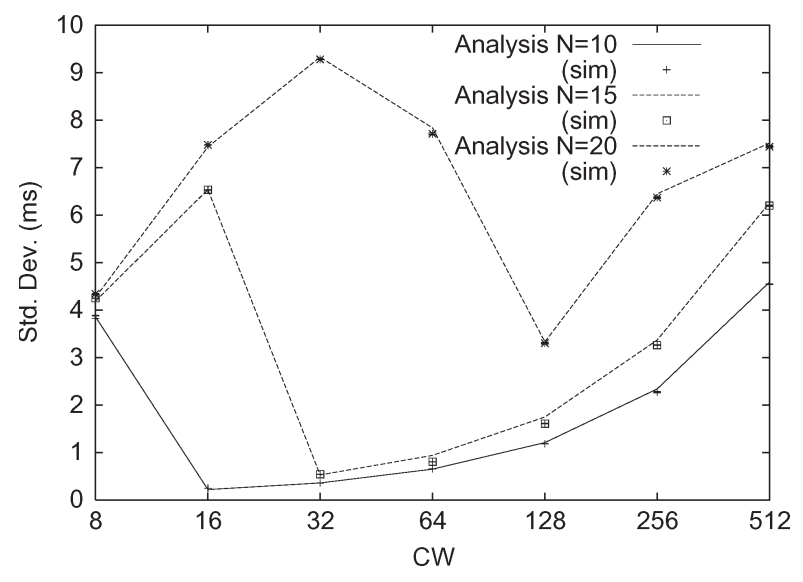

Fig. 7. Validation of the model for the standard deviation of the delay under voice traffic and different $C W$ configurations.

We observe from Fig. 6 that the evolution of the delay versus $C W$ shows a nonmonotonous behavior. Indeed, there is, at first, a steep decrease in the delay, reaching the minimum value, and then there is a slow increase. This is caused because, with small $C W$ values, there are many collisions in the WLAN, which cause congestion. When using larger $C W$, collisions take place less frequently, and the WLAN moves out of a congested situation; the steep decrease corresponds to this change from congestion to out of congestion. Then, after reaching the minimum value, there is a "graceful degradation" of the delay, which is caused by the use of larger back-off counters than needed to prevent congestion. ${ }^{10}$

3) Voice and Data Traffic: Next, we validate the model for the case of a WLAN operating with both data and voice traffic. The validation is performed using two ACs, both with the same number of stations.

- The first group (voice stations) transmits 80-B packets every $10 \mathrm{~ms}$.

- The second group (data stations) transmits according to a Poisson process with an average rate of $500 \mathrm{~kb} / \mathrm{s}$ and the packet lengths derived from the measurements in [38].

For validating our model, we perform the following experiments.

- First, we perform an experiment to validate the analysis of the differentiating effect of the AIFS parameter. To this aim, both ACs have the same contention window configuration $C W_{\min }=32, m=5$. Regarding the $A_{i}$ parameter, the voice $\mathrm{AC}$ is always configured with $A_{i}=0$, whereas for the configuration of the data $\mathrm{AC}$, we use two different values: $A_{i}=1$ (small differentiation), and $A_{i}=5$ (large differentiation).

- Similarly, we assess whether our model captures the differentiating effect of the $C W$ parameter by means of the following configuration: $A_{i}=0$ for the two ACs and $C W_{\min }=16, m=1$ for voice, whereas for data traffic, we use $C W_{\min }=32, m=4$ in one case and $C W_{\min }=$ $64, m=4$ in the other case.

\footnotetext{
${ }^{10}$ For a detailed analysis of this behavior, see [21].
}

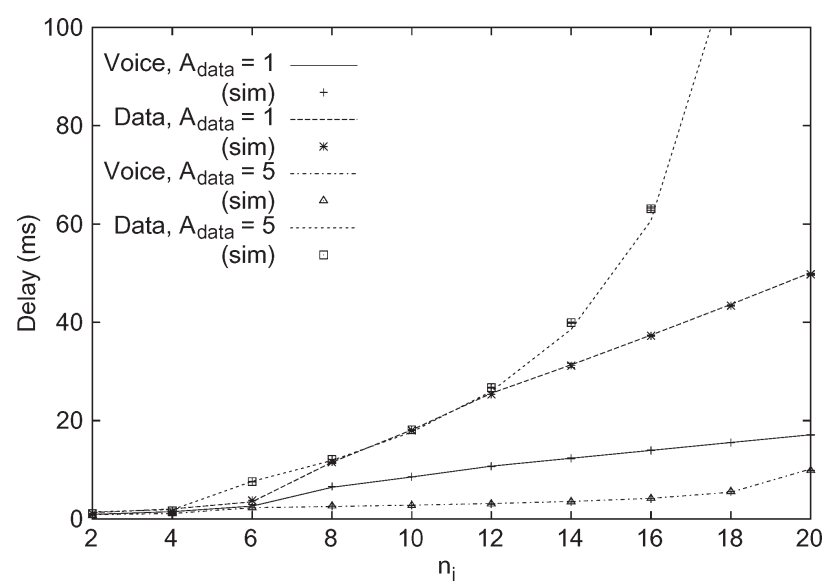

Fig. 8. Validation of the average delay model for a mixed scenario with voice and data stations and different $A I F S$ differentiation.

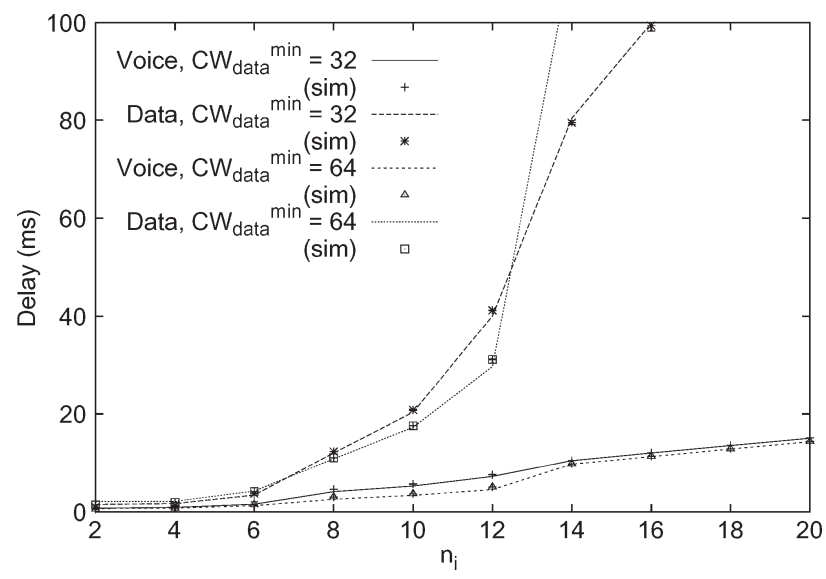

Fig. 9. Validation of the average delay model for a mixed scenario with voice and data stations and different $C W$ differentiation.

The results for the average delay are shown in Figs. 8 and 9. As the results from the analytical model closely follow the simulation values, we conclude that the proposed model is also valid for this case. It is worth remarking the degree of service differentiation that the $A I F S$ and $C W$ parameters provide: For the case of $A I F S$, the differentiation is strong only when there is enough traffic on the WLAN (i.e., $n_{i}$ is relatively large). On the other hand, the $C W$ parameter provides a larger level of differentiation.

The evaluation of the analysis of the standard deviation of the delay is depicted in Figs. 10 and 11. As in the previous case, the model closely follows the simulation results, which confirms the validity of our analysis for this performance metric as well. We further observe that, compared with the average delay, the standard deviation is more sensitive to the increase in the load.

4) Mixed Traffic: We finally validate our model for the more general case, with up to four traffic classes of different characteristics, which we name "voice," "video," "data," and "background," respectively.

- In the first AC (voice), 80-B packets are generated every $10 \mathrm{~ms}$.

- In the second AC (video), we model video traffic with a variable bit-rate source sending variable-size packets at 


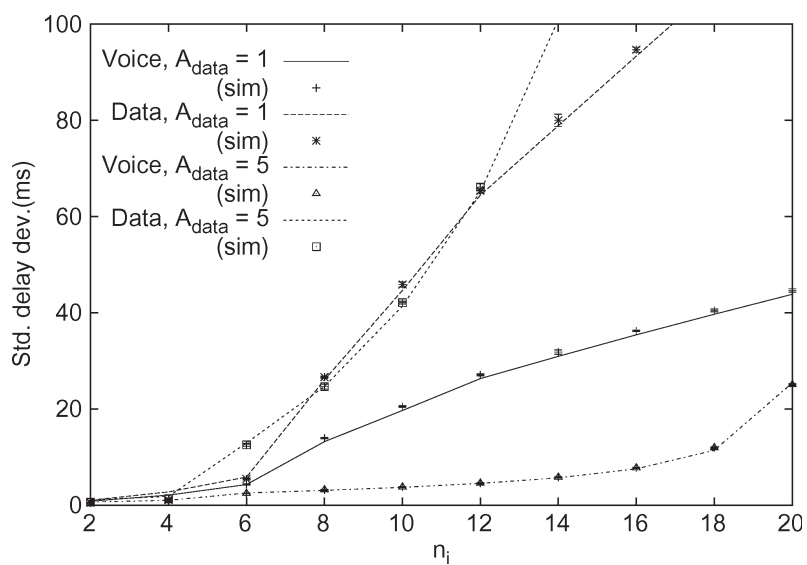

Fig. 10. Validation of the model of the standard deviation of the delay for a mixed scenario with voice and data stations and different $A I F S$ configurations.

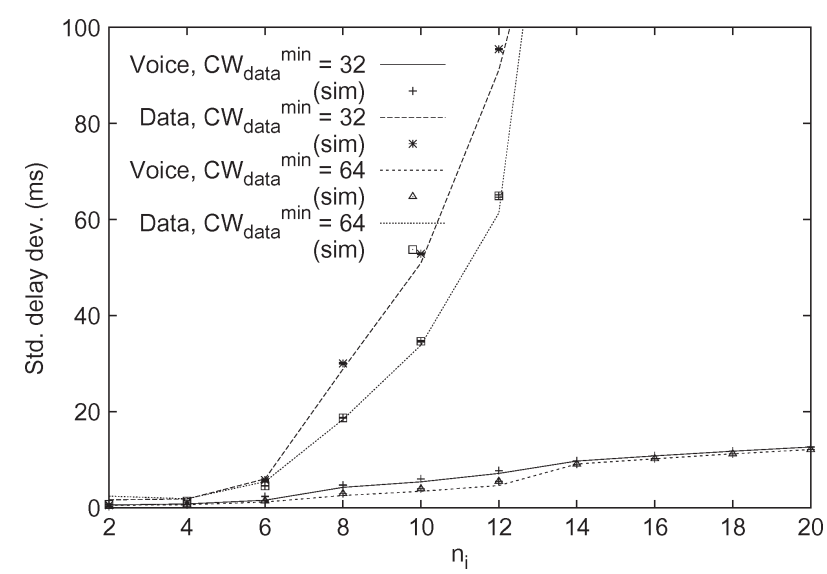

Fig. 11. Validation of the model of the standard deviation of the delay for a mixed scenario with voice and data stations and different $C W$ configurations.

TABLE III

EDCA CONFIGURATION

\begin{tabular}{|l|r|r|r|}
\cline { 2 - 4 } \multicolumn{1}{c|}{} & $A_{i}$ & \multicolumn{1}{c|}{$C W_{i}^{\text {min }}$} & \multicolumn{1}{c|}{$C W_{i}^{\max }$} \\
\hline voice & 0 & 8 & 16 \\
\hline video & 0 & 16 & 32 \\
\hline data & 1 & 32 & 1024 \\
\hline background & 5 & 32 & 1024 \\
\hline
\end{tabular}

a constant interarrival time. The average bit rate of the source is set equal to $250 \mathrm{~kb} / \mathrm{s}$, and the packet length distribution is taken from the video traffic measurements in [39].

- In the third AC (data), stations always have a packet that is ready for transmission, modeling the behavior of a data transfer. Packet sizes are taken from the data traffic measurements in [38].

- In the fourth AC (background), stations always have 1000-B packets that are ready for transmission.

The configuration of each $\mathrm{AC}$ is derived from the recommendations given in the 802.11e standard for 802.11b (see Table III). Experiments are performed for a varying number of stations per AC (each AC has $n_{i}$ stations). Figs. 12 and 13 plot the average and the standard deviation of the delay, respectively. The validation of the throughput model is depicted in Fig. 14.

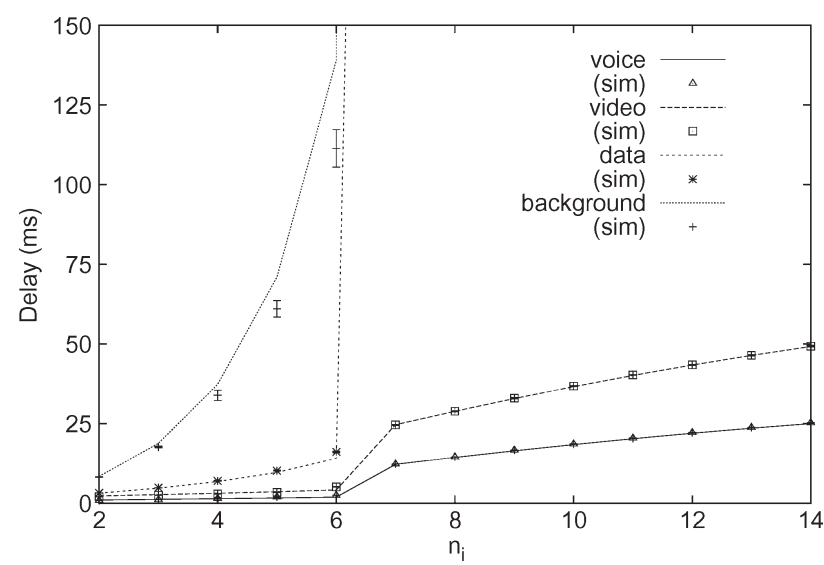

Fig. 12. Validation of the average delay model for a scenario with four ACs configured according to the standard recommended values.

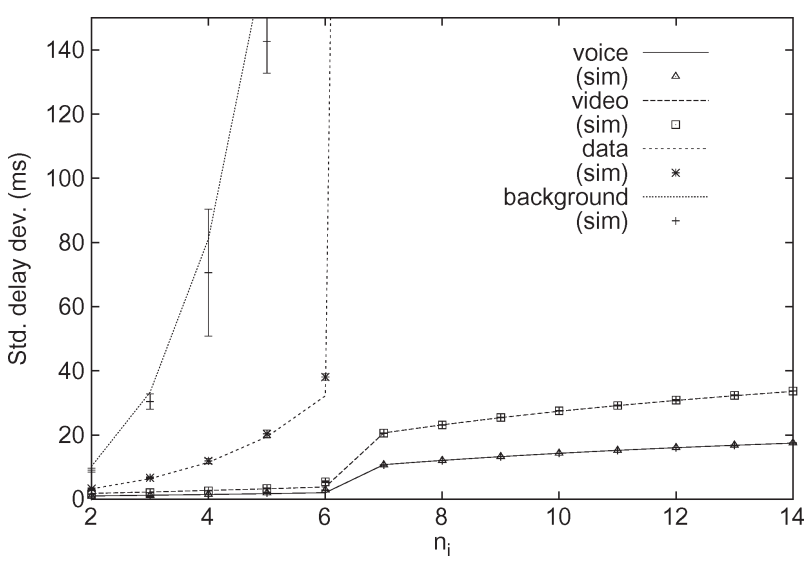

Fig. 13. Validation of the model for the standard deviation of the delay for a scenario with four ACs configured according to the standard recommended values.

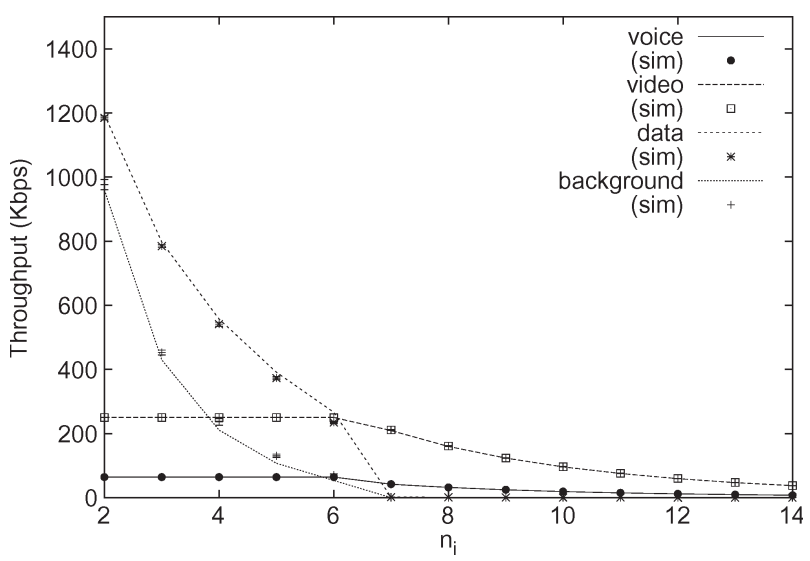

Fig. 14. Validation of the throughput model for a scenario with four ACs configured according to the standard recommended values.

We observe from the figures that EDCA is effective in providing service differentiation. Both in terms of the throughput and the delay, higher priority ACs always perform better than lower priority ones. Furthermore, higher priority ACs also saturate later: AC 3 (data) saturates for $n_{i}>4$, whereas ACs 1 
and 2 (voice and video) saturate for $n_{i}>6$ (AC 4 is, by definition, always saturated). Beyond this saturation point, the throughput of all ACs gradually decreases with $n_{i}$, whereas the delay increases drastically. For all cases, the analytical results match the simulations remarkably well, confirming the accuracy of our model.

\section{Optimal Configuration}

Here, we present an algorithm to find the optimal configuration of the EDCA parameters under a general scenario with multiple real-time and data ACs. The objectives of our algorithm are given as follows.

1) Meet the requirements of the real-time traffic. More specifically, the configuration should provide real-time stations with the required throughput and delay guarantees.

2) Maximize the admissibility in the network. Specifically, we aim to admit as many real-time stations as possible while satisfying the previous objective.

3) Maximize the throughput that is received by the data traffic while meeting the previous two objectives. For throughput allocation, we use the common weighted max-min fair allocation criterion [40]-[42]. This maximizes the minimum $r_{i} / w_{i}$ in the system, with $r_{i}$ being the throughput that is allocated to entity $i$ and $w_{i}$ being the entity's weight. In our case, the entities are the WLAN stations, and the allocated throughput is the saturation throughput of a station.

\section{A. Considerations for Optimal Configuration}

Before proceeding with the design of our algorithm, we make the following considerations on the configuration of some of the EDCA parameters. This simplifies the design of the algorithm and reduces its computational cost.

The considerations for the data $A C s$ are the following.

- From (1), we have that $\tau_{i}$ can be adjusted as a function of two parameters, i.e., $C W_{i}^{\min }$ and $m_{i}$. As a consequence, we have one degree of freedom when setting these parameters to obtain the desired $\tau_{i}$. Following this, we fix $m_{i}=0$. Then, by substituting $m_{i}$ with 0 in (1), we compute the $C W_{i}^{\text {min }}$ value that leads to $\tau_{i}^{o p t}$ as follows:

$$
C W_{i}=\frac{2}{\tau_{i}^{o p t}}-1 .
$$

- $A_{i}=A_{d} \forall i$ : Following the proof in [7], the throughput of the data stations is maximized when they all use the same $A_{i}$ setting.

- $T X O P=1$ packet. This ensures that a station transmitting data sends only one packet upon accessing the channel and, thus, reduces the delay that is inflicted on real-time traffic.

For the case of real-time ACs, we make the following considerations.

- $A_{i}=0$ : The optimal setting for this parameter is its minimum possible value, namely, $A I F S=D I F S$, as otherwise, additional time is unnecessarily lost after every transmission.
- $C W_{j}^{\min }=C W_{j}^{\max }=C W_{j}$ : When the number of stations in the channel is unknown, $C W_{\max }$ is typically set larger than $C W_{\min }$ so that after a collision, the $C W$ increases, and thus, the probability of a new collision is reduced. However, this is not necessary in our case, as the number of stations is known, and therefore, their $C W_{\min }$ can be directly configured for optimal operation. In addition, if we set $C W_{\max }$ larger than $C W_{\min }$, the delay of the packets that suffer one or more collisions drastically grows, which harms jitter performance.

- $T X O P_{j}=T X O P_{\max }$ : Considering the strict delay requirements of real-time traffic, the EDCA parameters will be chosen such that the transmission queues of the stations almost never grow to more than one packet (in particular, this holds for the configurations that we later propose). In the eventual case that queues grow above one packet, it is desirable that, upon accessing the channel, all waiting packets are transmitted to minimize their delay. To achieve this, we set the TXOP parameter to its maximum allowed value.

Following the above considerations, our algorithm provides the configuration for the following set of parameters that are left open: the $A_{i}$ configuration for the data stations, the $C W_{i}$ parameters for each of the real-time ACs, and the $C W_{i}$ for each data AC.

\section{B. Optimal Configuration Algorithm}

The proposed algorithm is based on a numerical search that we perform over the $\tau_{i}$ of one data $\mathrm{AC}$, which we take as reference. In each step of the search, given the value of the $\tau_{i}$ of this reference AC, we need to compute the $\tau_{j}$ of the other ACs. To obtain the $\tau_{j}$ of the other data ACs, we apply the max-min fair allocation criterion to the throughput expression given in (7), which yields [7]

$$
\frac{\tau_{i}\left(1-\tau_{j}\right)}{\tau_{j}\left(1-\tau_{i}\right)}=\frac{w_{i}}{w_{j}} .
$$

Once the $\tau_{j}$ 's of each data AC are known, the other $\tau_{l}$ 's can be obtained as follows. Neglecting the probability of a drop due to reaching the maximum retry limit, we have $r_{i} \approx$ $\rho_{i}$. Furthermore, by applying (7) to $r_{l} / r_{k}$ and making the approximation $p\left(s_{l}\right) / p\left(s_{k}\right) \approx \tau_{l} / \tau_{k}$, we obtain

$$
\frac{\tau_{l}}{\tau_{k}} \approx \frac{\rho_{l} / l_{l}}{\rho_{k} / l_{k}}
$$

where, hereafter, we will denote the right-hand side of the above equation by $K_{l}$.

With the above, we can derive a third-order equation to approximately calculate $\tau_{k}$ of a reference real-time AC, given all $\tau_{i}$ 's of the data ACs. This third-order equation is obtained from setting the output rate of a station of $\mathrm{AC} k$ equal to its input rate, i.e.,

$$
r_{k}=\rho_{k}
$$

where $r_{k}$ is computed by making the following simplification to the expression of (7): We distinguish two types of slots, i.e., the ones where only real-time stations can transmit and the 
ones where data stations can transmit, and then compute the numerator and the denominator of (7) by conditioning them to these two types of slots. This yields

$$
r_{k}=\frac{p\left(\Delta_{\text {real }}\right) p\left(s_{k} \mid \Delta_{\text {real }}\right)+p\left(\Delta_{\text {data }}\right) p\left(s_{k} \mid \Delta_{\text {data }}\right)}{p\left(\Delta_{\text {real }}\right) T_{\text {slot }, r}+p\left(\Delta_{\text {data }}\right) T_{\text {slot }, d}} l_{k}
$$

where $p\left(\Delta_{\text {real }}\right)$ is the probability that, in a slot time, only realtime ACs can transmit, $p\left(\Delta_{\text {data }}\right)$ is the probability that data stations can also transmit, $p\left(s_{k} \mid \Delta_{\text {real }}\right)$ and $p\left(s_{k} \mid \Delta_{\text {data }}\right)$ are the success probabilities of a station of AC $k$ for each of the two cases, and $T_{\text {slot, } r}$ and $T_{\text {slot, } d}$ are the average slot durations, respectively.

The probability $p\left(\Delta_{\text {real }}\right)$ is computed as follows. We first calculate the exact expression for the probability of being in a state in which only real-time ACs can transmit, and then, we perform a first-order approximation of the Taylor expansion of this expression. The result is the following:

$$
\begin{aligned}
p\left(\Delta_{\text {real }}\right) \approx & \frac{A_{d}\left(1-p\left(e \mid \Delta_{\text {data }}\right)\right.}{A_{d}\left(1-p\left(e \mid \Delta_{\text {data }}\right)\right)+1} \\
& +\frac{\left(1-p\left(e \mid \Delta_{\text {data }}\right)\right) A_{d}^{2}}{2\left(\left(1-p\left(e \mid \Delta_{\text {data }}\right) A_{d}+1\right)^{2}\right.} \tau_{k} \\
& +\frac{\left(1+p\left(e \mid \Delta_{\text {data }}\right)\right) A_{d} \sum_{l \in \Delta_{\text {real }}} n_{l} K_{l}}{2\left(\left(1-p\left(e \mid \Delta_{\text {data }}\right) A_{d}+1\right)^{2}\right.} \tau_{k}
\end{aligned}
$$

where $\Delta_{\text {real }}$ is the set of the real-time ACs, and $p\left(e \mid \Delta_{\text {data }}\right)$ is the probability that a slot in which all ACs can transmit is empty, i.e.,

$$
p\left(e \mid \Delta_{\text {data }}\right)=\prod_{k}\left(1-\tau_{k}\right) .
$$

$p\left(\Delta_{\text {data }}\right)$ is simply computed as

$$
p\left(\Delta_{\text {data }}\right)=1-p\left(\Delta_{\text {real }}\right) .
$$

The probability $p\left(s_{k} \mid \Delta_{\text {real }}\right)$ corresponds to the probability that a station of $\mathrm{AC} k$ transmits and that no other real-time station transmits, i.e.,

$$
\begin{aligned}
p\left(s_{k} \mid \Delta_{\text {real }}\right) & =\tau_{k}\left(1-\tau_{k}\right)^{n_{k}-1} \prod_{l \in \Delta_{\text {real }} \backslash k}\left(1-\tau_{l}\right)^{n_{l}} \\
& \approx \tau_{k}\left(1-\left(n_{k}-1\right) \tau_{k}-\sum_{l \in \Delta_{\text {real } \backslash k}} n_{l} K_{l} \tau_{k}\right)
\end{aligned}
$$

By considering that the probability that no other station transmits is approximately $p\left(e \mid \Delta_{\text {data }}\right)$, the probability $p\left(s_{k} \mid \Delta_{\text {data }}\right)$ corresponds to the probability that a station of AC $k$ transmits and that no other station, i.e., real-time or data, transmits, i.e.,

$$
p\left(s_{k} \mid \Delta_{\text {data }}\right) \approx \tau_{k} p\left(e \mid \Delta_{\text {data }}\right) .
$$

Finally, we can calculate $T_{\text {slot }, r}$ and $T_{\text {slot }, d}$ as a secondorder expression in $\tau_{k}$ by considering the different lengths of the transmissions that we can have in a slot time and the corresponding probabilities.
Based on the above analysis, our optimal configuration mechanism is described by Algorithm 1 and is summarized as follows.

- Given a reference data AC $i$ and a reference real-time AC $k$, a search is performed on all $A_{i}$ 's values specified in the standard (line 4). For each $A_{i}$ value, a golden section search is performed on the $\tau_{i}$ to maximize the throughput allocation criterion (line 5).

- For each value of $\tau_{i}$, the $\tau_{j}$ of the remaining data ACs is computed with (11) according to the allocation criterion (line 7).

- Next, the transmission probability $\tau_{k}$ of the reference realtime AC is computed with (13) (line 9), and from this, the remaining $\tau_{l}$ 's of the other real-time ACs are then computed by applying (12) (line 11).

- With all the $\tau$ 's, we proceed to compute the $C W$ values that guarantee delay performance to real-time stations. Following the explanations in [21], there is a range of $\mathrm{CW}$ values that provide the desired QoS performance. To compute this range of $\mathrm{CW}$ values, we use the delay analysis of Lemmas 3 and 4 to obtain the configurations that lead to the desired delay performance. With the alreadycomputed $\tau_{i}$ 's and the setting $C W_{\min }=C W_{\max }$, this can be efficiently done using (8) and (9).

- From all the $C W$ values, we choose the maximum one for each AC (line 14) since, following the discussion in [21], these are the ones that lead to a WLAN operating as far as possible from instability.

- We next check that the values of $C W$ obtained in the previous step satisfy the requirement that, even in the cases where the real-time stations become saturated, their throughput in saturation $r_{j}$ (sat) is larger than their input rate $\rho_{j}$ (line 18) since, following [43], this guarantees that the proposed configuration is indeed stable. If this condition is not met, then this configuration is not further considered in the search.

- Next, $\tau$ 's are used to compute the weighted rate $r_{i} / w_{i}$ of each data AC (line 23). Note that the golden section search of line 5 maximizes the minimum of these values. Therefore, if the current configuration provides better performance than the ones previously evaluated in the search, it is saved (lines 25-30).

- Finally, once the search ends, the best configuration is returned through the EDCA parameters (line 37). If the search provides no configuration, this means that there exists no configuration that satisfies the sources' requirements, and therefore, the request that triggered this search has to be rejected.

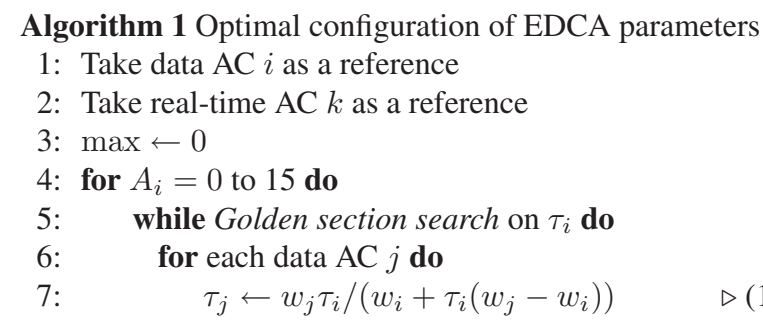


8: $\quad$ end for

9: $\quad$ Compute $\tau_{k}$

10: for each real-time $\mathrm{AC} j$ do

11:

12:

13:

14:

15:

$16:$

17:

18:

19: The

$$
\tau_{l} \leftarrow \tau_{k} K
$$

end for

for each real-time AC $j$ do

Compute $C W_{j}$ to fulfill

the delay requirement

$\triangleright$ Lemmas 3 and 4

\section{end for}

for each real-time AC $j$ do

$$
\text { if } r_{j}(s a t)<\rho_{j} \text { then }
$$

corresponds to saturation.

20:

21:

22:

23:

24:

configuration

else

for each data AC $j$ do

\section{end for}

Compute $r_{j} / w_{j}$

if $\min \left\{\left(r_{i} / w_{i}\right)\right\}>\max$ then

$\triangleright$ Save

$$
\max \leftarrow \min \left\{\left(r_{i} / w_{i}\right)\right\}
$$

$A_{\max } \leftarrow A_{i}$

$\tau_{\max }^{\text {data }} \leftarrow \tau^{\text {data }}$

$C W_{\max }^{\text {real-time }} \leftarrow C W^{\text {real-time }}$

end if

end if

end for

end while

3: end for

34: $C W^{\text {data }} \leftarrow 2 / \tau_{\max }^{\text {data }}-1$

35: return $A_{i}, C W^{\text {data }}, C W^{\text {real-time }}$

\section{Optimal Configuration Validation}

Here, we validate our optimal configuration algorithm by means of simulations for different traffic scenarios. More specifically, we assess through simulations the performance of the configuration resulting from our algorithm and compare it against the best performance obtained by performing an exhaustive search over the EDCA parameters.

1) Data Traffic: We first assess the performance of our algorithm for a scenario where only data stations are present. We consider a scenario where four ACs with $n_{i}$ stations each always have a 1500-B frame that is ready for transmission. In these circumstances, and with no real-time traffic in the WLAN, the only relevant metric of performance is the maximum $\min \left(r_{i} / w_{i}\right)$, according to the max-min fair allocation criterion. Results are shown in Table IV for $n_{i}=\{2,10\}$ stations per $\mathrm{AC}$ and different throughput allocation weights $w_{i}$ 's. They show that the configuration algorithm maximizes throughput performance as the gain obtained when using the exhaustive search is negligible.

2) Voice Traffic: Next, we evaluate the performance of our algorithm for voice traffic. We validate the algorithm by comparing the performance of our configuration ( $\left.C W_{\text {algorithm }}\right)$ against the result of performing an exhaustive search over the $C W_{\min }$ space and choosing the best $C W_{\min }$ value, i.e., $C W_{\text {exhaustive. }}$ We perform this experiment for three dif-
TABLE IV

ALGORITHM VALIDATION FOR DATA TRAFFIC SCENARIO

\begin{tabular}{|c|c|c|c|c|r|r|}
\hline$n_{i}$ & $w_{1}$ & $w_{2}$ & $w_{3}$ & $w_{4}$ & $\min \left(r_{i} / w_{i}\right)$ & $\begin{array}{r}\min \left(r_{i} / w_{i}\right) \\
\text { exhaustive } \\
\text { search }\end{array}$ \\
\hline \hline \multirow{2}{*}{2} & 1 & 2 & 3 & 4 & 212.21 & 212.52 \\
& 1 & 3 & 5 & 7 & 124.20 & 124.66 \\
& 1 & 4 & 7 & 10 & 88.12 & 88.41 \\
& 1 & 5 & 9 & 13 & 66.25 & 66.74 \\
\hline \multirow{3}{*}{10} & 1 & 2 & 3 & 4 & 41.79 & 41.85 \\
& 1 & 3 & 5 & 7 & 24.81 & 24.96 \\
& 1 & 4 & 7 & 10 & 18.62 & 18.71 \\
& 1 & 5 & 9 & 13 & 13.02 & 13.29 \\
\hline
\end{tabular}

\begin{tabular}{|c|c|c|c|c|c|c|c|c|}
\hline 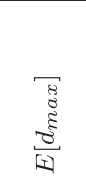 & $\begin{array}{l}\tilde{Z} \\
\tilde{\tilde{D}} \\
\tilde{E} \\
\tilde{b}\end{array}$ & $\approx$ & 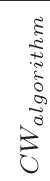 & 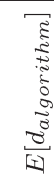 & 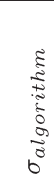 & 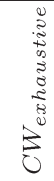 & 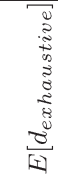 & 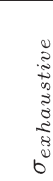 \\
\hline \multirow{3}{*}{$5 \mathrm{~ms}$} & \multirow{3}{*}{$5 \mathrm{~ms}$} & 10 & 314 & 4.95 & 2.78 & 317 & 4.99 & 2.82 \\
\hline & & 15 & 225 & 4.91 & 2.87 & 229 & 4.99 & 2.92 \\
\hline & & 20 & 118 & 4.72 & 3.02 & 125 & 4.99 & 3.25 \\
\hline \multirow{3}{*}{$5 \mathrm{~ms}$} & \multirow{3}{*}{$2.5 \mathrm{~ms}$} & 10 & 274 & 4.35 & 2.43 & 281 & 4.45 & 2.49 \\
\hline & & 15 & 186 & 4.07 & 2.36 & 196 & 4.28 & 2.49 \\
\hline & & 20 & 89 & 3.65 & 2.48 & 91 & 4.31 & 2.49 \\
\hline \multirow{3}{*}{$2.5 \mathrm{~ms}$} & \multirow{3}{*}{$2.5 \mathrm{~ms}$} & 10 & 145 & 2.45 & 1.32 & 148 & 2.49 & 1.35 \\
\hline & & 15 & 104 & 2.32 & 1.29 & 111 & 2.47 & 1.39 \\
\hline & & 19 & 66 & 2.29 & 1.42 & 72 & 2.49 & 1.54 \\
\hline
\end{tabular}

TABLE V

Algorithm VAlidation for Voice TrafFic ScEnARio

ferent quality criteria, ranging from a more stringent requirement $\left(E\left[d_{\max }\right], \sigma_{\max } \leq 2.5 \mathrm{~ms}\right)$ to a more relaxed one $\left(E\left[d_{\max }\right], \sigma_{\max } \leq 5 \mathrm{~ms}\right)$ [21]. Simulation results, which are presented in Table $\mathrm{V}$, show that 1) the proposed configuration is always very close to the one obtained from the exhaustive search, and 2) our algorithm admits as many voice calls as the exhaustive search while meeting the desired quality criteria.

3) Voice and Data Traffic: To validate the proposed algorithm for a scenario in which the WLAN operates under both data and voice traffic, we perform the following experiment. We consider two ACs, both with the same number of stations and the following characteristics.

- The first AC transmits 80-B packets every $10 \mathrm{~ms}$. We consider two different delay requirements for this AC: 1) $E[d], \sigma_{d} \leq 2.5 \mathrm{~ms}$, and 2) $E[d], \sigma_{d} \leq 5 \mathrm{~ms}$.

- The second AC models the behavior of a data transfer by always having a 1000-B packet that is ready for transmission.

Again, we compare the results from our configuration against those provided by the best configuration found by means of an exhaustive search over the $C W_{\min }$ of the data and voice ACs, and the $A_{i}$ parameter of the data AC. The results are shown in Table VI: With the proposed configuration, the quality criteria are always met, and the throughput obtained by data stations is very close to that provided by the configuration resulting from the exhaustive search. We, therefore, conclude that the proposed configuration algorithm maximizes the performance of the WLAN.

4) Mixed Traffic: Finally, to validate the proposed algorithm under the most generic scenario, we consider a WLAN with the four ACs defined in Section III-D4, each of them with the same number of stations $n_{i}$. For the real-time ACs, we consider the 
TABLE VI

Algorithm VAlidation for Data AND Voice TrafFic Scenario

\begin{tabular}{|c|c|c|c|c|r|}
\hline$n_{i}$ & $\begin{array}{c}E\left[d_{\max }\right], \\
\sigma_{d_{\max }}\end{array}$ & $E[d]$ & $\sigma_{d}$ & $r_{\text {data }}$ & $\begin{array}{r}r_{\text {data }} \\
\text { exhaustive } \\
\text { search }\end{array}$ \\
\hline \hline 5 & & 2.47 & 2.22 & 971.62 & 973.29 \\
10 & 2.5 & 2.49 & 2.37 & 319.04 & 324.23 \\
15 & & 2.47 & 2.45 & 113.85 & 117.14 \\
\hline 5 & & 4.85 & 4.30 & 974.62 & 976.83 \\
10 & 5 & 4.83 & 4.37 & 324.33 & 327.14 \\
15 & & 4.69 & 4.25 & 113.11 & 115.44 \\
\hline
\end{tabular}

TABLE VII

Algorithm VALidation FOR MiXed TrafFic SCENARio

\begin{tabular}{|c|r|r|r|r|r|r|}
\hline$n_{i}$ & $E\left[d_{1}\right]$ & $\sigma_{d, 1}$ & $E\left[d_{2}\right]$ & $\sigma_{d, 2}$ & $\min \left(r_{i} / w_{i}\right)$ & $\begin{array}{r}\min \left(r_{i} / w_{i}\right) \\
\text { exhaustive } \\
\text { search }\end{array}$ \\
\hline \hline 2 & 4.91 & 3.48 & 19.81 & 13.09 & 793.21 & 793.93 \\
4 & 4.96 & 3.55 & 19.78 & 13.00 & 304.34 & 304.82 \\
6 & 5.00 & 3.59 & 19.88 & 13.35 & 144.17 & 144.57 \\
8 & 5.00 & 3.59 & 19.54 & 13.04 & 64.87 & 65.15 \\
10 & 4.74 & 3.45 & 18.81 & 12.73 & 18.24 & 18.34 \\
\hline
\end{tabular}

following delay requirements: $E\left[d_{1}\right], \sigma_{d, 1} \leq 5 \mathrm{~ms}$ and $E\left[d_{2}\right]$, $\sigma_{d, 2} \leq 20 \mathrm{~ms}$ and, for the data ACs, the following weights: $w_{3}=2$, and $w_{4}=1$.

The throughput and delay results obtained with the proposed algorithm are given in Table VII. The results validate the proposed algorithm since they satisfy all the requirements.

- In all scenarios, the average and the standard deviation of the delay obtained with our configuration are below the desired values, which shows that the configuration meets the required delay guarantees.

- Furthermore, an exhaustive search has been conducted, which has shown that no other configuration can admit more stations while satisfying the delay requirements. This proves that the configuration maximizes the admissibility region by admitting as many stations as possible.

- Finally, by comparing the throughput performance obtained through exhaustive search against our results, we conclude that we also maximize the $\min \left(r_{i} / w_{i}\right)$ for data ACs.

\section{Comparison Against Other Approaches}

We next compare the performance of the configuration resulting from our algorithm against the following approaches.

- two other available approaches for the configuration of the EDCA parameters, namely, the standard recommended set of values [2] and the recent proposal in [26];

- the adaptive configuration schemes in [27], [31], and [32], which aim to provide QoS guarantees in EDCA WLANs. It is worth noting that the approaches in [31] and [32] require introducing changes to the 802.11e standard, which challenges their practical use.

The scenario that we choose for this comparison is the mixed traffic scenario of Section IV-C4 since this is the most complete of the scenarios used in the validation of the algorithm. Table VIII gives the average delay of voice and video flows (in milliseconds), as well as the total throughput given to data stations (in kilobits per second) resulting from our algorithm and from the other five mentioned approaches.

From the results given in the table, we conclude that our algorithm clearly outperforms the other proposals since 1) with our approach, real-time stations always see their delay guarantees satisfied; 2) our approach provides data stations with a substantially larger throughput than any other approach meeting the delay requirements ${ }^{11}$; and 3 ) it also provides a much larger admissibility region. In particular, with our approach, we can admit up to $n_{i}=10$ stations, while none of the other proposals can admit more than $n_{i}=6$ stations (i.e., our approach can admit at least $66 \%$ more stations). The reason for this is that the other approaches are based on heuristics that do not guarantee optimal performance, in contrast to ours, which is based on an analytical model that guarantees optimal performance.

\section{E. Implementation Considerations}

We assess the computational cost of the algorithm by measuring the number of floating-point operations (flops) required by a MATLAB implementation to execute it. For all the presented experiments, the algorithm requires approximately 90 kflops. Assuming a WLAN access point with a 10-Mflops/s CPU, it would take $9 \mathrm{~ms}$ to perform an admission control decision, which is fully acceptable in a realistic scenario. We conclude from this experiment that the computational complexity of the proposed algorithm is sufficiently low to allow its practical use in today's hardware platforms.

\section{CONCLUSION}

As the EDCA mechanism of $802.11 \mathrm{e}$ becomes widely available, the need for a configuration algorithm to tune the MAC parameters and boost WLAN performance arises. We have shown that a proper configuration of EDCA can lead to performance gains of $66 \%$ over the standard recommended values. We believe that these gains represent a strong motivation for the deployment of EDCA WLANs to efficiently use the scarce wireless medium.

In this paper, we have presented an algorithm to configure an EDCA WLAN that achieves a twofold objective: 1) It maximizes the admissibility region of real-time traffic, and 2) it optimizes the throughput performance of data traffic. To build this algorithm, we have presented the most comprehensive analysis of EDCA performance to date. This analysis, as proven by exhaustive simulations, can accurately model throughput and delay performance of real-time and nonreal-time traffic.

We have used this analysis to design an optimal configuration algorithm for EDCA. In contrast to previous work, which is typically heuristic or measurement-based, ours is a mathematically supported mechanism that tunes the EDCA parameters to maximize performance. By means of the analytical model,

\footnotetext{
${ }^{11}$ Although, for the $n_{i}=10$ case, data stations receive a larger throughput with [31] than with our configuration, voice and video stations suffer much larger delays. Additionally, they also suffer a drop rate above 20\% (not shown in the table), which results in our approach actually providing better total throughput performance.
} 
TABLE VIII

COMPARISON AgAinst OTHER APPROACHES

\begin{tabular}{|c|c|c|c|c|c|c|c|c|c|c|c|c|c|c|c|c|c|c|}
\hline \multirow[b]{2}{*}{$n_{i}$} & \multicolumn{3}{|c|}{ Our algorithm } & \multicolumn{3}{|c|}{ Standard } & \multicolumn{3}{|c|}{ [26] } & \multicolumn{3}{|c|}{ [31] } & \multicolumn{3}{|c|}{ [32] } & \multicolumn{3}{|c|}{ [27] } \\
\hline & $d_{v o}$ & $d_{v i}$ & $r_{\text {data }}$ & $d_{v o}$ & $d_{v i}$ & $r_{\text {data }}$ & $d_{v o}$ & $d_{v i}$ & $r_{\text {data }}$ & $d_{v o}$ & $d_{v i}$ & $r_{\text {data }}$ & $d_{v o}$ & $d_{v i}$ & $r_{\text {data }}$ & $d_{v o}$ & $d_{v i}$ & $r_{\text {data }}$ \\
\hline 2 & 4.9 & 19.8 & 4759 & 0.9 & 2.1 & 4728 & 0.4 & 1.9 & 3808 & $\overline{5.9}$ & $\overline{6.6}$ & 2127 & 1.1 & 2.6 & 2990 & 2.1 & $\overline{5.0}$ & 3724 \\
\hline 4 & 4.9 & 19.7 & 3652 & 1.5 & 2.9 & 3474 & 0.6 & 2.5 & 2576 & 9.9 & 11.2 & 2327 & 1.9 & 5.4 & 2752 & 4.9 & 12.2 & 1713 \\
\hline 6 & 5.0 & 19.8 & 2595 & 2.5 & 5.1 & 2093 & 0.8 & 4.9 & 1478 & 9.9 & 19.5 & 1868 & 4.0 & 10.0 & 1616 & 18.7 & 41.7 & 104 \\
\hline 8 & 5.0 & 19.5 & 1556 & 14.5 & 28.9 & 9 & 1.3 & 32.4 & 1 & 10.0 & 26.6 & 894 & 11.6 & 32.2 & 347 & 33.4 & 76.4 & 65 \\
\hline 10 & 4.7 & 18.8 & 547 & 18.6 & 36.6 & 4 & 1.4 & 46.9 & 0 & 15.9 & 27.0 & 831 & 14.9 & 42.3 & 278 & 50.6 & 48.5 & 146 \\
\hline
\end{tabular}

we have derived an efficient algorithm whose complexity is well suited for low computation capacity devices and can be implemented in realistic scenarios. We have shown that the performance of our algorithm is almost identical to the one obtained through exhaustive numerical searches.

\section{APPENDIX}

Lemma 1: The $\tau_{i}$ of a nonsaturated $\mathrm{AC}$ is given by $\tau_{i}^{\text {nonsat }}=\frac{\rho_{i}\left(1-p\left(c_{i}\right)^{R+1}\right)\left(p(s) T_{s}+p(e) T_{e}+p(c) T_{c}\right)}{l_{i}\left(1-\tau_{i}\right)^{n_{i}-1} \sum_{k=A_{i}}^{A} p\left(\Delta_{k}\right) \prod_{j \in \Delta_{k} \backslash i}\left(1-\tau_{j}\right)^{n_{j}}}$.

Proof: According to Section III-A, a station of a nonsaturated AC sees that all the traffic it sends served either because its packets are successfully transmitted or because they are discarded when reaching the retry limit due to suffering $R+1$ collisions. Hence, the following holds:

$$
\rho_{i}\left(1-p\left(c_{i}\right)^{R+1}\right)=r_{i}
$$

where $r_{i}$ is the throughput that is experienced by a station of $A C i$, which is given by (7), $\rho_{i}$ is its average sending rate, and $p\left(c_{i}\right)^{R+1}$ corresponds to the probability that a packet of this station is discarded upon reaching the retry limit.

To prove the lemma, we need to derive the different variables in (7). The probability $p(e)$ is, by definition, $p\left(e \mid t_{0}\right)$, as all slot times are 0-slot times. This has already been computed in Section III-B. To compute the rest of the variables in (7), we proceed as follows. First, let us define $p\left(t_{k}\right)$ as the probability that a slot time is a $k$-slot time. Since a slot time is a $k$-slot time if and only if the previous slot time is a $(k-1)$-slot time and it is empty, which occurs with a probability $p\left(e \mid t_{k-1}\right)$, this probability can be expressed as

$$
p\left(t_{k}\right)=p\left(t_{k-1}\right) p\left(e \mid t_{k-1}\right) \text {. }
$$

Starting from $p\left(t_{0}\right)=1$ (which holds by definition) and recursively applying the above, it follows that

$$
p\left(t_{k}\right)=\prod_{j=0}^{k-1} p\left(e \mid t_{j}\right)
$$

The probability that a random slot time contains a success of a given station of $A C i$ can be computed (by applying the total probability theorem) as

$$
p\left(s_{i}\right)=\sum_{k=A_{i}}^{A} p\left(\Delta_{k}\right) p\left(s_{i} \mid \Delta_{k}\right)
$$

where $p\left(s_{i} \mid \Delta_{k}\right)$ is the probability that a slot time in which this set of ACs may transmit contains a success of a given station of $A C i$.

A slot time is allowed for transmission to the set $\Delta_{k}$ (with $k<A)$ if the slot time is a $k$-slot time but not a $(k+1)$-slot time. ${ }^{12}$ For $k=A$, we have that in an $A$-slot time, all ACs are allowed to transmit. Thus

$$
p\left(\Delta_{k}\right)= \begin{cases}p\left(t_{k}\right)-p\left(t_{k+1}\right), & k<A \\ p\left(t_{A}\right), & k=A\end{cases}
$$

The probability $p\left(s_{i} \mid \Delta_{k}\right)$ corresponds to the case when the considered station transmits and no other station of set $\Delta_{k}$ does, i.e.,

$$
p\left(s_{i} \mid \Delta_{k}\right)=\tau_{i}\left(1-\tau_{i}\right)^{n_{i}-1} \prod_{j \in \Delta_{k} \backslash i}\left(1-\tau_{j}\right)^{n_{j}} .
$$

The probability that a slot time contains a success can be computed as the sum of the individual success probabilities, i.e.,

$$
p(s)=\sum_{i \in \Delta_{A}} n_{i} p\left(s_{i}\right)
$$

where, with our definition of $A, \Delta_{A}$ denotes the set of all ACs. The probability that a slot time contains a collision can be obtained from

$$
p(c)=1-p(e)-p(s) .
$$

The average duration of a success $T_{s}$ can be computed by summing the different possible durations weighted by their probabilities, i.e.,

$$
T_{s}=\sum_{i \in \Delta_{A}} \frac{n_{i} p\left(s_{i}\right)}{p(s)} T_{s, i}
$$

where $T_{s, i}$ is the average duration of a success of a station of $\mathrm{AC} i$, which is calculated according to the following expression given by [7]:

$$
T_{s, i}=T_{\mathrm{PLCP}}+\frac{H+l_{i}}{C}+S I F S+T_{\mathrm{PLCP}}+\frac{A C K}{C}+D I F S
$$

where $T_{\mathrm{PLCP}}$ is the physical-layer convergence protocol preamble and header transmission time, $H$ is the MAC overhead (header and FCS), ACK is the size of the acknowledgment frame, and $C$ is the channel bit rate.

\footnotetext{
${ }^{12}$ Note that a slot time that is a $k$-slot time but not a $(k+1)$-slot time is preceded by exactly $k$ empty slot times, and therefore, only the ACs with $A_{i} \leq$ $k$ (i.e., the ACs of set $\Delta_{k}$ ) may transmit in such a slot time.
} 
To compute the average duration of a collision $T_{c}$, we note that this is given by the largest packet length involved. Following this, we can compute $T_{c}$ by summing the possible collision durations weighted by their probabilities, i.e.,

$$
T_{c}=\sum_{l \in L} \frac{p(c, t=l)}{p(c)} T_{c}^{l}
$$

where $p(c, t=l)$ is the probability that a slot time contains a collision in which the length of the longest packet involved is equal to $l, T_{c}^{l}$ is the duration of this collision, and $L$ is the set of packet lengths.

$T_{c}^{l}$ is computed as (see [7])

$$
T_{c}^{l}=T_{\mathrm{PLCP}}+\frac{H+l}{C}+E I F S
$$

and $p(c, t=l)$ is computed, applying the total probability theorem, as

$$
p(c, t=l)=\sum_{k=0}^{A} p\left(\Delta_{k}\right) p\left(c, t=l \mid \Delta_{k}\right)
$$

where $p\left(c, t=l \mid \Delta_{k}\right)$ is the probability that, given that only stations of set $\Delta_{k}$ may transmit, a slot time contains a collision in which the longest packet involved is of length $l$.

To obtain $p\left(c, t=l \mid \Delta_{k}\right)$, we sweep along all the stations that may transmit and compute the probability that 1) the considered station transmits a packet of length $l$, and 2) some other station transmits a packet but with length that is no longer than $l$. Let us define $S_{k}$ as the set of stations of $\Delta_{k}$ and $p\left(t_{j}=l\right)$ as the probability that the length of a transmission from station $j$ is $l$. Then

$$
p\left(c, t=l \mid \Delta_{k}\right)=\sum_{j \in S_{k}} \tau_{j} p\left(t_{j}=l\right) p\left(t x \leq l \mid S_{k}, j\right)
$$

where $p\left(t x \leq l \mid S_{k}, j\right)$ accounts for the probability that there is at least one transmission from the set $S_{k}$ (without station $j$ ) but of a size that is less than or equal to $l$. To compute this probability, we calculate the probability that no station transmits a packet that is longer than $l$ and subtract from this the probability that no station transmits. In particular, for the computation of the first term, we index all the stations and refer with $S_{k, j}$ to the set of stations of $S_{k}$ with an index that is smaller than $j$; then, we compute the probability that stations of $S_{k, j}$ do not transmit a packet that is longer than or equal to $l$ and the probability that stations with higher index than $j$ do not transmit a packet that is longer than $l,{ }^{13}$ i.e.,

$$
\begin{aligned}
& p\left(t x \leq l \mid S_{k}, j\right)=\prod_{m \in S_{k, j}}\left(1-\tau_{m} p\left(t_{m} \geq l\right)\right) \\
& \times \prod_{m \in S_{k} \backslash S_{k, j} \cup j}\left(1-\tau_{m} p\left(t_{m}>l\right)\right)-\prod_{m \in S_{k} \backslash j}\left(1-\tau_{m}\right) .
\end{aligned}
$$

\footnotetext{
${ }^{13}$ The distinction in (35) between the stations with indexes that are smaller and larger than $j$ is made to avoid counting more than once the event when two or more stations transmit a packet of length $l$.
}

Finally, expressing $r_{i}$ as a function of the variables computed in (22)-(35) and substituting these into (20) yield

$$
\begin{aligned}
\rho_{i}\left(1-p\left(c_{i}\right)^{R+1}\right) & =\tau_{i}\left(1-\tau_{i}\right)^{n_{i}-1} l_{i} \\
& \times \frac{\sum_{k=A_{i}}^{A} p\left(\Delta_{k}\right) \prod_{j \in \Delta_{k} \backslash i}\left(1-\tau_{j}\right)^{n_{j}}}{\left(p(s) T_{s}+p(e) T_{e}+p(c) T_{c}\right)} .
\end{aligned}
$$

The proof follows.

Lemma 2: The average throughput $r_{i}$ that a station from $A C i$ experiences is given by

$$
r_{i}=\frac{l_{i} \sum_{k=A_{i}}^{A} p\left(\Delta_{k}\right) p\left(s_{i} \mid \Delta_{k}\right)}{p(s) T_{s}+p(c) T_{c}+p(e) T_{e}} .
$$

Proof: We compute the throughput $r_{i}$ following [34]: We divide the average payload information transmitted by $A C i$ in

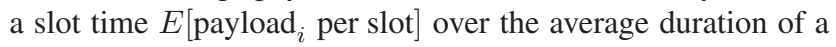
slot time $E$ [slot length], i.e.,

$$
r_{i}=\frac{E\left[\text { payload }_{i} \text { per slot }\right]}{E[\text { slot length }]} .
$$

The average payload information transmitted by $A C i$ is given by

$$
E\left[\text { payload }_{i} \text { per slot }\right]=l_{i} p\left(s_{i}\right)
$$

while the average length of a slot time is given by

$$
E[\text { slot length }]=p(s) T_{s}+p(c) T_{c}+p(e) T_{e}
$$

where the probabilities and average durations have already been derived in the proof of Lemma 1. By combining the above equations, we obtain

$$
r_{i}=\frac{l_{i} p\left(s_{i}\right)}{p(s) T_{s}+p(c) T_{c}+p(e) T_{e}} .
$$

The proof follows.

Lemma 3: The average delay experienced by a nondropped packet of a station of $A C i$ is given by

$$
\begin{aligned}
d_{i} & =\frac{1}{\sum_{j=0}^{R}\left(1-p\left(c_{i}\right)\right) p\left(c_{i}\right)^{j}} \sum_{j=0}^{R}\left(1-p\left(c_{i}\right)\right) p\left(c_{i}\right)^{j} \\
& \times\left(j T_{c, i}+T_{s, i}+\sum_{r=0}^{j}\left(T_{\text {inter_tx,k}}^{i}+B_{i, r}\left(T_{\text {slot }, k}^{i}+T_{\text {inter }, k}^{i}\right)\right)\right) .
\end{aligned}
$$

Proof: To compute the average delay of a nondropped packet $d_{i}$, we use the total probability theorem as follows:

$$
d_{i}=\frac{\sum_{j=0}^{R}\left(1-p\left(c_{i}\right)\right) p\left(c_{i}\right)^{j} d_{i, j}}{\sum_{j=0}^{R}\left(1-p\left(c_{i}\right)\right) p\left(c_{i}\right)^{j}}
$$

where $d_{i, j}$ is defined as the average delay of a station of $A C i$ in case the frame suffers $j$ retries. This delay is computed as 
(see Fig. 3)

$$
d_{i, j}=\sum_{r=0}^{j}\left(T_{\text {inter_tx,k}}^{i}+B_{i, r}\left(T_{\text {slot }, k}^{i}+T_{\text {inter }, k}^{i}\right)\right)+j T_{c, i}+T_{s, i} .
$$

To complete the proof of the lemma, we need to compute the components of (44). $T_{s, i}$ is given by (30). $B_{i, r}$ is computed using the following given in [13]:

$$
B_{i, r}=\frac{C W_{i}^{\min } 2^{\min \left(m_{i}, r\right)}-1}{2} .
$$

$T_{c, i}$ is computed by applying the total probability theorem

$$
T_{c, i}=\frac{\sum_{k=A_{i}}^{A} T_{c, i, k} p\left(\Delta_{k}\right)}{\sum_{k=A_{i}}^{A} p\left(\Delta_{k}\right)}
$$

where $T_{c, i, k}$ is the average duration of a collision in which a station of AC $i$ is involved when only the ACs of set $\Delta_{k}$ may transmit. This is computed as follows:

$$
T_{c, i, k}=\frac{\sum_{l \in L} T_{c}^{l} p\left(c_{i}, t=l \mid S_{k}\right)}{\sum_{l \in L} p\left(c_{i}, t=l \mid S_{k}\right)}
$$

where $p\left(c_{i}, t=l \mid S_{k}\right)$ is the probability that a slot time in which a station of AC $i$ transmits and the stations of set $S_{k}$ may transmit contains a collision of length $l$. This is computed by distinguishing between the case that a station of AC $i$ transmits a frame of size $l$ [with probability $p\left(t_{i}=l\right)$ ] or smaller [with probability $p\left(t_{i}<l\right)$ ], i.e.,

$$
\begin{aligned}
& p\left(c_{i}, t=l \mid S_{k}\right) \\
&=p\left(t_{i}=l\right) \cdot\left(\prod_{m \in S_{k} \backslash i}\left(1-\tau_{m} p\left(t_{m}>l\right)\right)-\prod_{m \in S_{k} \backslash i}\left(1-\tau_{m}\right)\right) \\
&+p\left(t_{i}<l\right)\left(\sum_{j \in S_{k} \backslash i} \tau_{j} p\left(t_{j}=l\right) \prod_{m \in S_{k, j} \backslash i}\left(1-\tau_{m} p\left(t_{m} \geq l\right)\right)\right. \\
&\left.\times \prod_{m \in S_{k} \backslash\left\{S_{k, j} \cup i, j\right\}}\left(1-\tau_{m} p\left(t_{m}>l\right)\right)\right) .
\end{aligned}
$$

$T_{\text {slot }, k}^{i}$ is computed as the sum of probabilities of success, empty, and collision multiplied by the average slot-time duration in each case, i.e.,

$$
\begin{aligned}
s T_{\mathrm{slot}, k}^{i}=p\left(s \mid S_{k}, i\right) & T_{s, k}^{i}+p\left(e \mid S_{k}, i\right) T_{e} \\
& +\left(1-p\left(s \mid S_{k}, i\right)-p\left(e \mid S_{k}, i\right)\right) T_{c, k}^{i}
\end{aligned}
$$

where $p\left(e \mid S_{k}, i\right)$ and $p\left(s \mid S_{k}, i\right)$ are the probabilities that a $k$-slot time in which the considered station does not transmit ${ }^{14}$

\footnotetext{
${ }^{14}$ The condition that the considered station does not transmit holds until the end of the proof.
}

is empty and contains a success, respectively, and $T_{s, k}^{i}$ and $T_{c, k}^{i}$ are the average slot-time durations of a success and a collision, respectively.

$T_{s, k}^{i}$ is computed by applying the total probability theorem, i.e.,

$$
T_{s, k}^{i}=\frac{\sum_{j=k}^{A} p\left(\Delta_{j}\right) \sum_{m \in \Delta_{j}} n_{m, i} p\left(s_{m} \mid \Delta_{j}, i\right) T_{s, m}}{p\left(s \mid S_{k}, i\right)}
$$

where $n_{m, i}=n_{m}-\delta_{i m}$ (the Kronecker function $\delta_{i m}$ accounts for the fact that the considered station does not transmit), $p\left(s_{m} \mid \Delta_{j}, i\right)$ is the probability that given the set $\Delta_{j}$ can transmit but station $i$ did not transmit, there is a success from $A C m$, i.e.,

$$
p\left(s_{m} \mid \Delta_{j}, i\right)=\tau_{m}\left(1-\tau_{m}\right)^{n_{m, i}-1} \prod_{j \in \Delta_{k} \backslash m}\left(1-\tau_{j}\right)^{n_{j, i}}
$$

and $p\left(s \mid S_{k}, i\right)$ is computed by adding the success probabilities of each AC, i.e.,

$$
p\left(s \mid S_{k}, i\right)=\sum_{j=k}^{A} p\left(\Delta_{j}\right) \sum_{m \in \Delta_{j}} n_{m, i} p\left(s_{m} \mid \Delta_{j}, i\right) T_{s, m} .
$$

$T_{c, k}^{i}$ is computed similarly to (50), i.e.,

$$
T_{c, k}^{i}=\frac{\sum_{j=k}^{A} \sum_{l \in L} T_{c}^{l} p\left(c, t=l \mid S_{j}, i\right) p\left(\Delta_{j}\right)}{\sum_{j=k}^{A} \sum_{l \in L} p\left(c, t=l \mid S_{j}, i\right) p\left(\Delta_{j}\right)}
$$

where

$$
p\left(c, t=l \mid S_{k}, i\right)=\sum_{j \in S_{k} \backslash i} \tau_{j} p\left(t_{j}=l\right) p\left(t x \leq l \mid S_{k}, i, j\right)
$$

is the probability of a collision of size $l$ in a $k$-slot, with $p\left(t x \leq l \mid S_{k}, i, j\right)$ being the probability that at least one station other than $i$ and $j$ transmits a frame of smaller than or equal to $l$, which is computed following (35). Finally, we compute $p\left(e \mid S_{k}, i\right)$ by applying a similar reasoning to (3), i.e.,

$$
p\left(e \mid S_{k}, i\right)=\frac{p\left(e \mid t_{k}\right)}{1-\tau_{i}} .
$$

$T_{\text {inter }, k}^{i}$ is computed as follows. If the given slot time is empty, which occurs with probability $p\left(e \mid S_{k}, i\right)$, then $T_{\text {inter }, k}^{i}=$ 0 . Otherwise, $T_{\text {inter }, k}^{i}$ is, by definition, equal to $T_{\text {inter_t } t x, k}^{i}$. Thus

$$
T_{\text {inter }, k}^{i}=\left(1-p\left(e \mid S_{k}, i\right)\right) T_{\text {inter_tx }, k}^{i} .
$$

The above relies on $T_{\text {inter_tx,k}}^{i}$, which is the time between a nonempty timeslot and the next $k$-slot. To compute it, we consider the number of $j$ empty slots that follow the transmission(s) and distinguish two cases: 1) when the number of $j$ empty timeslots is equal to $k$ and, therefore, the time until the next $k$-slot is composed of exactly $k$ empty slot times and 2) when $j<k$ and, therefore, the time is composed of $j$ empty slot times, a nonempty slot where only stations from 
$S_{j}$ can transmit, and an additional time that is, by definition, $T_{\text {inter_t } t, k}^{i}$. This way

$$
\begin{aligned}
T_{\text {inter_tx }, k}^{i}= & \prod_{j=0}^{k} p\left(e \mid S_{j}, i\right) k T_{e} \\
& +\sum_{j=0}^{k-1}\left(\prod_{l=0}^{j} p\left(e \mid S_{l}, i\right)\left(1-p\left(e \mid S_{j+1}, i\right)\right)\right) \\
& \cdot\left(j T_{e}+T_{\text {slot_tx,j }}^{i}+T_{\text {inter_tx,k }}^{i}\right)
\end{aligned}
$$

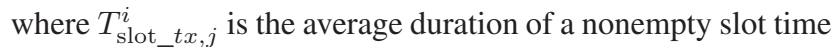
preceded by a nonempty $k$-slot time followed by $j$ empty slot times, which is computed as the probability that such a slot time contains a collision multiplied by the average duration in this case, plus the probability that it contains a success multiplied by the corresponding average duration, i.e.,

$$
\begin{aligned}
& T_{\text {slot }, t x, j}^{i} \\
& =\left(1-\frac{\sum_{m \in S_{j}} n_{m} \tau_{m}\left(1-\tau_{m}\right)^{n_{m}-1} \prod_{p \in S_{j} \backslash m}\left(1-\tau_{p}\right)^{n_{p}}}{1-\prod_{m \in S_{j}}\left(1-\tau_{m}\right)^{n_{m}}}\right) \\
& \quad \cdot T_{c, j}^{i}+\frac{\sum_{m \in S_{j}} n_{m} \tau_{m}\left(1-\tau_{m}\right)^{n_{m}-1} \prod_{p \in S_{j} \backslash m}\left(1-\tau_{p}\right)^{n_{p}}}{1-\prod_{m \in S_{j}}\left(1-\tau_{m}\right)^{n_{m}}} T_{s, j}^{i} .
\end{aligned}
$$

Equations (57) and (58) can be reduced to a first-order equation on $T_{\text {inter_tx, } k}^{i}$, from which we can isolate this term and then derive $T_{\text {inter, } k}^{i}$. By combining all the above equations, we obtain the expression for the average delay given by the lemma, as well as the computation of all the terms of this expression. The proof follows.

Lemma 4: The standard deviation of the delay is given by

$$
\sigma_{d_{i}}^{2}=\frac{\sum_{j=0}^{R}\left(1-p\left(c_{i}\right)\right) p\left(c_{i}\right)^{j} E\left[\left(d_{i, j}\right)^{2}\right]}{\sum_{j=0}^{R}\left(1-p\left(c_{i}\right)\right) p\left(c_{i}\right)^{j}}-\left(d_{i}\right)^{2} .
$$

Proof: To compute the standard deviation of the delay, i.e., $\sigma_{d_{i}}^{2}$, we use the following statistical relationship between the average and the second-order moment:

$$
\sigma_{d_{i}}^{2}=E\left[\left(d_{i}\right)^{2}\right]-\left(d_{i}\right)^{2} .
$$

We already have computed $d_{i}$ in (8), and therefore, the remaining challenge is to compute the second order of the average delay, i.e., $E\left[\left(d_{i}\right)^{2}\right]$. To this aim, we proceed similarly to (43), i.e.,

$$
E\left[\left(d_{i}\right)^{2}\right]=\frac{\sum_{j=0}^{R}\left(1-p\left(c_{i}\right)\right) p\left(c_{i}\right)^{j} E\left[\left(d_{i, j}\right)^{2}\right]}{\sum_{j=0}^{R}\left(1-p\left(c_{i}\right)\right) p\left(c_{i}\right)^{j}} .
$$

To compute $E\left[\left(d_{i, j}\right)^{2}\right]$, we rewrite $d_{i, j}$ in (44) as

$$
d_{i, j}=T_{s, i}+j T_{c, i}+j T_{\text {inter_tx }, k}^{i}+d_{b o}^{i, j}
$$

where $d_{b o}^{i, j}$ is the average time spent in back-off counter decrements for $A C i$ in case of $j$ retries, i.e.,

$$
\begin{aligned}
d_{b o}^{i, j}= & \sum_{n} p(b o=n \mid A C i, j \text { ret } x) \\
& \cdot \underbrace{\left(T_{\text {slot }, k}^{i}+T_{\text {inter }, k}^{i}\right)+\cdots+\left(T_{\text {slot }, k}^{i}+T_{\text {inter }, k}^{i}\right)}_{n \text { times }}
\end{aligned}
$$

where $p(b o=n \mid A C i, j$ ret $x)$ is the probability that the total number of back-off counter decrements after $j$ retries is $n$. This is computed through $j$ convolutions of the different uniform distributions that the station may use to compute its back-off counter, i.e.,

$$
\begin{array}{rl}
p(\text { bo }=n \mid A C i, j \text { ret } x) & =U\left(0, C W_{i}^{\min }-1\right) * \cdots \\
* & U\left(0,2^{\min \left(j, m_{i}\right)} C W_{i}^{\min }-1\right) .
\end{array}
$$

With the above, we proceed as follows to compute $E\left[\left(d_{i, j}\right)^{2}\right]$ :

$$
E\left[\left(d_{i, j}\right)^{2}\right]=\left(d_{i, j}\right)^{2}+\sigma_{d_{i, j}}^{2}
$$

where $\sigma_{d_{i, j}}^{2}$ is given by the sum of the variances of the components of (62). With our assumption that slot-time durations are independent

$$
\sigma_{d_{i, j}}^{2}=j \sigma_{T_{c, i}}^{2}+\sigma_{T_{s, i}}^{2}+(j+1) \sigma_{T_{\text {inter_tx,k }}^{i}}^{2}+\sigma_{d_{b o}^{i, j}}^{2} .
$$

Given the previous expressions, the computation of $E\left[\left(d_{i}\right)^{2}\right]$ (and, therefore, the analysis of the standard deviation of the delay) is laborious but straightforward, as it basically involves redoing the analysis of the average delay but computing secondorder moments and variances. By combining all the above equations, we obtain the expression for the average delay given by the lemma, as well as the computation of all the terms of this expression. The proof follows.

\section{REFERENCES}

[1] Amendment to Standard for Information Technology. LAN/MAN Specific Requirements-Part 11: Wireless LAN Medium Access Control (MAC) and Physical Layer (PHY) specifications: Medium Access Control (MAC) Enhancements for Quality of Service (QoS), IEEE Std. 802.11 WG, Nov. 2005.

[2] IEEE Standard for Information Technology-Telecommunications and Information Exchange Between Systems-Local and Metropolitan Area Networks-Specific Requirements-Part 11: Wireless LAN Medium Access Control (MAC) and Physical Layer (PHY) Specifications, IEEE Std. 802.11-2007 (Revision of IEEE Std. 802.11-1999), 12, 2007.

[3] S. Mangold, S. Choi, G. Hiertz, O. Klein, and B. Walke, "Analysis of IEEE 802.11e for QoS Support in Wireless LANs," Wireless Commun., vol. 10 , no. 6 , pp. 40-50, Dec. 2003.

[4] S. Choi, J. Prado, and S. Shankar, "IEEE 802.11e contention-based channel access (EDCF) performance evaluation," in Proc. IEEE ICC, 2003, pp. 1151-1156.

[5] A. Grilo and M. Nunes, "Performance evaluation of IEEE 802.11e," in Proc. PIMRC, Lisboa, Portugal, Sep. 2002, pp. 511-517.

[6] D. Gu and J. Zhang, "A new measurement-based admission control method for IEEE802.11 wireless local area networks," in Proc. IEEE PIMRC, Sep. 2003, pp. 2009-2013.

[7] A. Banchs and L. Vollero, "Throughput analysis and optimal configuration of 802.11e EDCA," Comput. Netw., vol. 50, no. 11, pp. 1749-1768, Aug. 2006. 
[8] J. W. Robinson and T. S. Randhawa, "Saturation throughput analysis of IEEE 802.11e enhanced distributed coordination function," IEEE J. Sel. Areas Commun., vol. 22, no. 5, pp. 917-928, Jun. 2004.

[9] Z. Kong, D. H. K. Tsang, B. Bensaou, and D. Gao, "Performance analysis of IEEE 802.11e contention-based channel access," IEEE J. Sel. Areas Commun., vol. 22, no. 10, pp. 2095-2106, Dec. 2004.

[10] V. Ramaiyan, A. Kumar, and E. Altman, "Fixed point analysis of single cell IEEE 802.11e WLANs: Uniqueness, multistability and throughput differentiation," in Proc. ACM SIGMETRICS, Jun. 2005, pp. 109-120.

[11] J. Hui and M. Devetsikiotis, "A unified model for the performance analysis of IEEE 802.11e EDCA,” IEEE Trans. Commun., vol. 53, no. 9, pp. 14981510, Sep. 2005.

[12] H. Zhu and I. Chlamtac, "Performance analysis for IEEE 802.11e EDCF service differentiation," IEEE Trans. Wireless Commun., vol. 4, no. 4, pp. 1779-1788, Jun. 2005.

[13] A. Banchs and L. Vollero, "A delay model for IEEE 802.11e EDCA," IEEE Commun. Lett., vol. 9, no. 6, pp. 508-510, Jun. 2005.

[14] H. Y. Hwang, S. J. Kim, D. K. Sung, and N.-O. Song, "Performance analysis of IEEE 802.11E EDCA with a virtual collision handler," IEEE Trans. Veh. Technol., vol. 57, no. 2, pp. 1293-1297, Mar. 2008.

[15] P. E. Engelstad and O. N. Osterbo, "Non-saturation and saturation analysis of IEEE 802.11e EDCA with starvation prediction," in Proc. 8th ACM MSWIM, Oct. 2005, pp. 244-233.

[16] G.-R. Cantieni, Q. Ni, C. Barakat, and T. Turletti, "Performance analysis of finite load sources in 802.11 b multirate environments," Comput. Commun., vol. 28, no. 10, pp. 1095-1109, Jun. 2005.

[17] J. W. Tantra, C. H. Foh, I. Tinnirello, and G. Bianchi, "Analysis of the IEEE 802.11e EDCA under statistical traffic," in Proc. ICC, Istanbul, Turkey, Jun. 2006, pp. 546-551

[18] K. Duffy, D. Malone, and D. Leith, "Modeling the 802.11 distributed coordination function in non-saturated conditions," IEEE Commun. Lett., vol. 9, no. 8, pp. 715-717, Aug. 2005.

[19] B. Li and R. Battiti, "Analysis of the 802.11 DCF with service differentiation support in non-saturation conditions," in Proc. 5th QofIS, Sep. 2004, pp. 64-73.

[20] P. Serrano, A. Banchs, and A. Azcorra, "A throughput and delay model for IEEE 802.11e EDCA under non saturation," Wireless Pers. Commun., vol. 43, no. 2, pp. 467-479, Oct. 2007.

[21] P. Serrano, A. Banchs, and J. Kukielka, "Optimal configuration of 802.11e EDCA under voice traffic," in Proc. IEEE GLOBECOM, 2007, pp. 5107-5111.

[22] Y. Ge, J. C. Hou, and S. Choi, "An analytic study of tuning systems parameters in IEEE 802.11e enhanced distributed channel access," Comput. Netw., vol. 51, no. 8, pp. 1955-1980, Jun. 2007.

[23] D. Gao, J. Cai, C. H. Foh, C.-T. Lau, and K. N. Ngan, "Improving WLAN VoIP capacity through service differentiation," IEEE Trans. Veh. Technol., vol. 57, no. 1, pp. 465-474, Jan. 2008.

[24] M. Narbutt and M. Davis, "Experimental tuning of AIFSN and CWmin parameters to prioritize voice over data transmission in $802.11 \mathrm{e}$ WLAN networks," in Proc. IWCMC, 2007, pp. 140-145.

[25] C. Cano, B. Bellalta, and M. Oliver, "Adaptive admission control mechanism for IEEE 802.11e WLANs," in Proc. IEEE 18th PIMRC, Sep. 2007, pp. 1-5.

[26] J. Lee, W. Liao, J.-M. Chen, and H.-H. Lee, "A practical QoS solution to voice over IP in IEEE 802.11 WLANs," IEEE Commun. Mag., vol. 47, no. 4, pp. 111-117, Apr. 2009.

[27] J. Freitag, N. L. S. da Fonseca, and J. F. de Rezende, "Tuning of 802.11e network parameters," IEEE Commun. Lett., vol. 10, no. 8, pp. 611-613, Aug. 2006.

[28] W.-T. Chen, "An effective medium contention method to improve the performance of IEEE 802.11," Wireless Netw., vol. 14, no. 6, pp. 769776, Dec. 2008

[29] K. A. Meerja and A. Shami, "Analysis of enhanced collision avoidance scheme proposed for IEEE 802.11e-enhanced distributed channel access protocol," IEEE Trans. Mobile Comput., vol. 8, no. 10, pp. 1353-1367, Oct. 2009.

[30] J.-C. Chen and K.-W. Cheng, "EDCA/CA: Enhancement of IEEE 802.11E EDCA by contention adaption for energy efficiency," IEEE Trans. Wireless Commun., vol. 7, no. 8, pp. 2866-2870, Aug. 2008.

[31] A. Nafaa and A. Ksentini, "On sustained QOS guarantees in operated IEEE 802.11 wireless LANs," IEEE Trans. Parallel Distrib. Syst., vol. 19, no. 8, pp. 1020-1033, Aug. 2008.

[32] Y. Xiao, F. H. Li, and B. Li, "Bandwidth sharing schemes for multimedia traffic in the IEEE 802.11e contention-based WLANs," IEEE Trans. Mobile Comput., vol. 6, no. 7, pp. 815-831, Jul. 2007

[33] F. Calí, M. Conti, and E. Gregori, "Dynamic tuning of the IEEE 802.11 protocol to achieve a theoretical throughput limit," IEEE/ACM Trans. Netw., vol. 8, no. 6, pp. 785-799, Dec. 2000.
[34] G. Bianchi, "Performance analysis of the IEEE 802.11 distributed coordination function," IEEE J. Sel. Areas Commun., vol. 18, no. 3, pp. 535-547, Mar. 2000.

[35] H. Wu, Y. Peng, K. Long, S. Cheng, and J. Ma, "Performance of reliable transport protocol over IEEE 802.11 wireless LAN: Analysis and enhancement," in Proc. IEEE INFOCOM, Jun. 2002, pp. 599-607.

[36] G. Grimmett and D. Stirzaker, Probability and Random Processes, 3rd ed. London, U.K.: Oxford Univ. Press, 2001.

[37] Information Technology-Telecommun. and Information Exchange Between Systems. Local and Metropolitan Area Networks. Specific Requirements. Part 11: Wireless LAN Medium Access Control (MAC) and Physical Layer (PHY) Specifications: High-Speed Physical Layer Extension in the 2.4 GHz Band, IEEE Std. 802.11 WG, Sep. 1999.

[38] K. Claffy, G. Miller, and K. Thompson, "The nature of the beast: Recent traffic measurements from an Internet backbone," in Proc. INET, Geneve, Switzerland, Jul. 1998.

[39] L. Muscariello, M. Mellia, M. Meo, R. L. Cigno, and M. Ajmone, "A simple Markovian approach to model Internet traffic at edge routers," COST279, Tech. Doc. TD(03)032, May 2003.

[40] D. Bertsekas and R. G. Gallager, Data Networks. Englewood Cliffs, NJ: Prentice-Hall, 1987.

[41] L. Massoulié and J. Roberts, "Bandwidth sharing: Objectives and algorithms," IEEE/ACM Trans. Netw., vol. 10, no. 3, pp. 320-328, Jun. 2002.

[42] N. H. Vaidya, P. Bahl, and S. Gupta, "Distributed fair scheduling in a wireless LAN," in Proc. Mobile Comput. Netw., 2000, pp. 167-178.

[43] P. Serrano, A. Banchs, T. Melia, and L. Vollero, "Performance anomalies of nonoptimally configured wireless LANs," in Proc. IEEE WCNC, Las Vegas, NV, Apr. 2006, pp. 920-925.

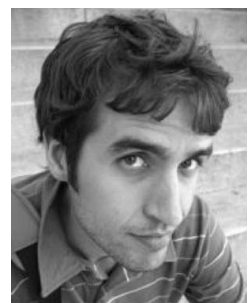

Pablo Serrano (M'09) received the Telecommunication Engineering and Ph.D. degrees from the University Carlos III of Madrid (UC3M), Leganés, Spain, in 2002 and 2006, respectively.

Since 2002, he has been with the Telematics Department, UC3M, where he is currently an Assistant Professor. In 2007, he was a Visiting Researcher with the Computer Network Research Group, University of Massachusetts, Amherst, which was partially supported by the Spanish Ministry of Education under a José Castillejo grant. He has over 20 scientific papers in peer-reviewed international journals and conferences. His current work focuses on performance evaluation of wireless networks.

Dr. Serrano serves as a Technical Program Committee member of several international conferences, including the IEEE Global Telecommunications Conference and the IEEE Conference on Computer Communications.

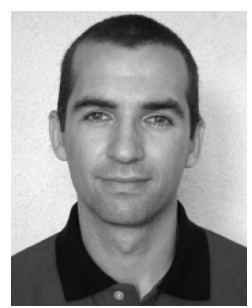

Albert Banchs (M'04) received the Telecommunications Engineering and $\mathrm{Ph} . \mathrm{D}$. degrees from the Polytechnical University of Catalonia, Barcelona, Spain, in 1997 and 2002, respectively.

In 1997, he was a Visiting Researcher with the International Computer Science Institute, Berkeley, CA. In 1998, he was with Telefonica I+D and, from 1998 to 2003, with NEC Europe Ltd., Heidelberg, Germany. Since 2003, he has been with the University Carlos III of Madrid, Leganés, Spain. Since 2009, he has been a Deputy Director with Instituto Madrileño de Estudios Avanzados (IMDEA) Networks, Leganés. He has authored over 50 publications in peer-reviewed journals and conferences. He is the holder of four patents (two of them granted).

Prof. Banchs has been a Guest Editor for two different issues: one special issue of IEEE Wireless Communications Magazine, and a different special issue of Elsevier Computer Networks. He has served on the Technical Program Committee (TPC) of a number of conferences and workshops, including the IEEE Conference on Computer Communications, the IEEE International Conference on Communications, and the IEEE Global Telecommunications Conference. He is the TPC Chair for European Wireless 2010. His Ph.D. thesis received the national award for Best Thesis on Broadband Networks. 


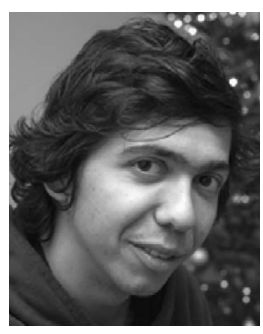

Paul Patras (S'08) received the Telecommunications Engineering degree in 2006 from the Technical University of Cluj-Napoca, Cluj-Napoca, Romania, and the M.Sc. degree in telematics engineering in 2008 from the University Carlos III of Madrid, Leganés, Spain, where he is currently working toward the Ph.D. degree.

Since 2007, he has been a Research Assistant with IMDEA Networks, Leganés. His current research interests include performance optimization in IEEE 802.11 wireless local area networks, adaptive medium-access control mechanisms, quality-of-service provisioning in wireless mesh networks, prototype implementation, and testbeds.

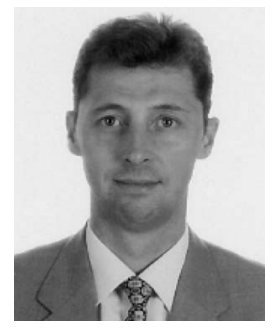

Arturo Azcorra (SM'02) received the M.Sc. degree in telecommunications engineering and the Ph.D. degree from the Universidad Politécnica de Madrid, Madrid, Spain, in 1986 and 1989, respectively, and the MBA degree from the Instituto de Empresa, Madrid, in 1993

He has recently been appointed the Director General for Technology Transfer and Entrepreneurial Development with the Spanish Ministry of Science and Innovation. As a result, he is on leave from his double appointment as a Full Professor (with Chair) with the Telematics Engineering Department, University Carlos III of Madrid, Leganés, Spain, and as the Director of IMDEA Networks, Leganés. He founded the not-for-profit institute in 2006 and has conducted his research activities there since its inception. He has published over 100 scientific papers in books, international magazines, and conferences.

Dr. Azcorra is a member of the Association for Computing Machinery Special Interest Group on Data Communication. He has participated in and directed 49 research and technological development projects, including the European Strategic Program on Research in Information Technology, R\&D in Advanced Communication for Europe, Advanced Communications Technologies and Services, and Information Society Technologies programs. He has coordinated the Content Distribution Network Research and Emerging Networking Experiments and Technologies European Networks of Excellence. $\mathrm{He}$ has served as a Program Committee Member for numerous international conferences, including several editions of the IEEE Conference Protocols for Multimedia Systems, Interactive Distributed Multimedia Systems, Quality of Future Internet Services, the Conference on Emerging Networking Experiments and Technologies, and the IEEE Conference on Computer Communications. 